\title{
7.
}

\section{Untersuchungen über die analytischen Facultäten.}

(Von dem Herrn Prof. Oettinger zu Freyburg im Br.)

(Fortsetzung der Abhandlung No. 1. im vorigen Heft.)

\section{I I.}

§. 16.

Bei der Fortsetzung dieser Untersuchungen nehmen wir nun hauptsächlich auf Anwendungen Rücksicht; wozu sich die bisher gefundenen Resultate benutzen lassen werden. Vor allem gewähren die in (§. 9.) aufgestellten Gleichungen eine weit verbreitete, bisher nicht gemachte Anwendung; welche hier gezeigt werden soll. Ueberall nämlich, wo die Summenausdrücke der Verbindungen mit und ohne Wiederholungen auftreten, finden auch die in (\$. 8. und 9.) aufgestellten Sätze ihre Geltung, und hiedurch eine Reihe von Problemen der Analysis ihre Auflösung, die zum Theil bis jetzt nicht, zum Theil nicht in dieser Allgemeinheit gegeben wurde. Schon Kramp hat darauf (Anal. d. réfr. Pg. 81.) aufmerksam gemacht, ohme jedoch die hergehörigen Gesetze aufzustellen.

Wir wollen zuerst die in ( $\$$. 8. und 9.) gefundenen Gesetze auf die Erhebung der Polynomien in Potenzen anwenden. Es ist bekanntlich

$$
\text { 1) } \boldsymbol{P}=\frac{1}{x} \log \frac{1}{1-x}=1+\frac{x}{2}+\frac{x^{2}}{3}+\frac{x^{3}}{4}+\ldots
$$

Nun ist, wie aus der Analysis bekannt:

$$
\begin{aligned}
& \text { 2) } P^{n}=\left(\frac{1}{x} \log \frac{1}{1-x}\right)^{n}=1+\frac{S C(1,2 \ldots n)^{1}}{n+1} x+\frac{S C(1,2 \ldots n+1)^{2}}{(n+1)^{2 \mid 1}} x^{2}+\frac{S C(1,2 \ldots . n+2)^{3}}{(n+1)^{3 / 1}} x^{3} \ldots \\
& \ldots+\frac{S C(1,2 \ldots . . n+r-1)^{r}}{(n+1)^{r \mid 1}} x^{r} \pm \ldots
\end{aligned}
$$

Werden die erforderlichen Werthe aus (10. \$. 9.) in (2) gesetzt und die nöthigen Ausscheidungen gemacht, so ergiebt sich: 
3) $\quad \boldsymbol{p}^{n}=1+\frac{n}{1^{2 \mid 1}} x+\frac{3(n+2)-1}{4.1^{3 \mid 1}} n x^{2}+\frac{(n+3)^{2 \mid-1} n}{2.24} x^{3}$

$$
\begin{aligned}
& +\frac{15(n+4)^{3}-30(n+4)^{2}+5(n+4)+2}{48.1^{511}} n x^{4} \\
& +\frac{3(n+5)^{4}-10(n+5)^{3}+5(n+5)^{2}+2(n+5)}{16.1^{6,1}} n \cdot x^{5}
\end{aligned}
$$

Hieraus findet sich unmittelbar, wenn $-n$ statt $n$ gesetzt wird:

$$
\text { 4) } \begin{aligned}
p^{-n}=1-\frac{n}{1^{|| 1}} & +\frac{3(n-2)+1}{4.1^{3 \mid 1}} n x^{2}-\frac{(n-3)^{2 \mid 1}}{2.1^{4 \mid 1}} n x^{3} \\
& +\frac{15(n-4)^{3}+30(n-4)^{2}+5(n-4)-2}{48.1^{5 \mid 1}} n x^{4} \\
& -\frac{3(n-5)^{4}+10(n-5)^{3}+5(n-5)^{2}-2(n-5)}{16.1^{6 \mid 1}} n x^{5}
\end{aligned}
$$

Aus diesem Ausdruck und aus (8. §. 9.) ergiebt sich:

5) $P^{-n}=1-\frac{S C^{\prime}(1,2, \ldots . n-1)^{1}}{n-1} x+\frac{S C^{\prime}(1,2, \ldots . n-2)^{2}}{(n-1)^{2 \mid 1}} x^{2}-\frac{S C^{\prime}(1,2, \ldots . n-3)^{2}}{(n-1)^{3 / 1}} x^{3}+\ldots$

$$
\ldots(-)^{r} \frac{s C^{u}(1,2, \ldots n-1)^{r}}{(n-1)^{r-1}} x^{r} \text {. }
$$

Aus (2. und 5.) ergiebt sich ein nicht zu übersehender Zusammenhang zwischen den Summenausdrücken der Verbindungen mit und ohne Wiederholungen, wenn sie bei Erhebung der Polynomien auf Potenzen benutzt werden. Aus (3. und 4.) erhält man folgende Entwicklungen für die Erhebung des vorliegenden Polynomiums zu einer Potenz mit gebrochenem Exponenten:

6) $\boldsymbol{p}^{\frac{n}{m}}=1+\frac{n x}{1^{2}} \frac{3(n+2 m)-m}{4.1^{3 / 1} m^{2}} n x^{2}+\frac{(n+3 m)^{21-m}}{2.1^{411} m^{3}} n x^{3}$

$$
\begin{aligned}
& +\frac{15(n+4 m)^{3}-30(n+4 m)^{2} m+5(n+4 m)^{2}+2 m^{3}}{48.1^{511} m^{4}} n x^{4} \\
& +\frac{3(n+5 m)^{4}-10(n+5 m)^{3} m+5(n+5 m)^{2} m^{2}-2(n+5 m) m^{3}}{16.1^{1 / 1} m^{5}} n x^{5}
\end{aligned}
$$

7) $\boldsymbol{P}^{-\frac{n}{m}}=1-\frac{n}{1^{2} \mid{ }^{1 /}} x+\frac{3(n-2 m)+m}{1^{4 / 1} m^{2}} n x^{2}-\frac{(n-3 m)^{2 \mid+m}}{2.1^{4 \mid 1} m^{3}} n x^{3}$

$$
\begin{aligned}
& +\frac{15(n-4 m)^{3}+30(n-4 m)^{2} m+5(n-4 m) m^{2}-2 m^{3}}{48.1^{511} m^{4}} n x^{4} \\
& +\frac{3(n-5 m)^{4}+10(n-5 m)^{3} m+5(n-5 m)^{2} m^{2}-2(n-5 m) m^{3}}{16.1^{6 / 1} m^{5}} n x^{6}
\end{aligned}
$$

Nun ist, wie ferner aus der Analysis bekannt: 
8) $\quad p^{n}=\left(1+\frac{x}{2}+\frac{x^{2}}{3}+\frac{x^{3}}{4}+\ldots .\right)^{n}$

$$
\begin{aligned}
= & \boldsymbol{P}^{\prime}(s n)^{n}+\boldsymbol{P}^{\prime}(s(n+1))^{n} x+\boldsymbol{P}^{\prime}(s(n+2))^{n} x^{2}+\ldots \boldsymbol{P}^{\prime}((s n+r))^{n} x^{\prime}+\ldots \\
& \left(1, \quad \frac{1}{2}, \quad \frac{1}{3}, \quad \frac{1}{4}, \ldots\right),
\end{aligned}
$$

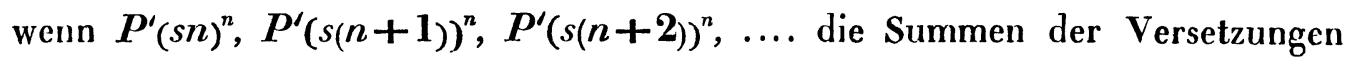
mit Wiederholungen in der $n$ ten Classe zu den Summen $n, n+1, n+2, \ldots$ aus den untergeschriebenen Elementen bezeichnen. Hieraus und aus (2) findet sich noch eine neue Art der Bildung der Verbindungen ohne Wiederholungen. Diesen Gleichungen zu Folge kann man nämlich die Verbindungen ohne Wiederholungen aus den Versetzungen mit Wiederholungen zu bestimmten Summen ableiten. Es ist

$$
\text { 9) } \frac{S C\left(1,2,3, \ldots .(n+r-1)^{r}\right.}{(n+1)^{r \mid 1}}=P^{\prime}\left(s(m+r) ; 1, \frac{1}{2}, \frac{1}{3}, \frac{1}{4}, \ldots\right)^{n}
$$

oder

10) $S C(1,2,3, \ldots n+r-1)^{r}=(n+1)^{r \mid 1} P^{\prime}\left(s(m+r) ; 1, \frac{1}{2}, \frac{1}{3}, \frac{1}{4}, \ldots\right)^{n}$.

$$
\text { §. } 17 .
$$

Eine weitere Anwendung ist die Erhebung des Polynomiums

$$
\text { 1) } P=x+\frac{x^{2}}{1.2}+\frac{x^{3}}{1.2 .3}+\frac{x^{4}}{1.2 .3 .4}+\ldots=e^{x}-1
$$

zur $n$ ten Potenz. Die Analysis giebt (S. d. Journ. 13ter Bd. S. 292 u. If. §. 47.),

2) $\boldsymbol{P}^{n}=\left(e^{x}-1\right)^{n}=S C^{\prime}(1,2, \ldots n)^{0} x^{n}+\frac{S C^{\prime}(1,2, \ldots . n)^{1} x^{n+1}}{n+1}+\frac{S C^{\prime}(1,2, \ldots . n)^{2} x^{n+2}}{(n+1)^{2 \mid 1}}+\ldots$

$$
\ldots \frac{S C^{\prime}(1,2, \ldots n)^{r} x^{n+r}}{(n+1)^{r \mid 1}}+\ldots
$$

Werden hier die angezeigten Werthe aus (§. 9.) eingeführt, so erhält man:

$$
\text { 3) } \begin{aligned}
\boldsymbol{p}^{n}=x^{n}\left(1+\frac{n}{1.2} x\right. & +\frac{3 n+1}{4.1^{3 \mid 1}} n x^{2}+\frac{n^{2 \mid 1}}{1^{2: 1} .1^{4 \mid 1}} n x^{3} \\
& +\frac{15 n^{3}+30 n^{2}+5 n-2}{48.1^{5 \mid 1}} n x^{4} \\
& +\frac{3 n^{4}+10 n^{3}+5 n-2 n}{16.1^{6 \mid 1}} n x^{5}
\end{aligned}
$$

Crelle's Journal f. d. M. Bd. XXXIII. Heft 2. 


$$
\begin{aligned}
& \text { 3) } P^{-n}=\frac{1}{x^{n}}\left(1-\frac{n}{2} x+\frac{3 n-1}{4.1^{3 \mid 1}} n x^{2}-\frac{n^{2 \mid 1}}{1^{2 \mid 1} .1^{4 \mid 1}} n x^{3}\right. \\
& +\frac{15 n^{3}-30 n^{2}+5 n+2}{48.1^{5 \mid 1}} n x^{4} \\
& -\frac{3 n^{4}-\frac{10 n^{3}+5 n^{2}+2 n}{16.16 \mid} n x^{5}}{16}
\end{aligned}
$$

Der besondere Fall, wenn in (4.) $n=1$ ist, giebt die Bernoullischen Zahlen. Aus (2. und 4.) ist also auch

5) $P^{-n}=\left(e^{x}-1\right)^{-n}=\frac{1}{x^{n}}\left(1-\frac{S C(1,2, \ldots . n-1)^{1}}{n-1} x+\frac{S C(1,2, \ldots n-1)^{2}}{(n-1)^{2 i-1}} x^{2}-\frac{S C(1,2, \ldots n-1)^{3}}{(n-1)^{3 !-1}} x^{3}+\ldots\right.$

$$
\left.\ldots(-1)^{r} \frac{S C(1,2, \ldots . n-1)^{r}}{(n-1)^{r \mid-1}} x^{r} \ldots . .\right)
$$

Für einen gebrochenen Exponenten ist

$$
\text { 6) } \begin{aligned}
\boldsymbol{p}^{\frac{n}{m}}=x^{\frac{n}{m}}\left(1+\frac{n}{2 m} x\right. & +\frac{3 n+m}{1^{4 \mid 1} m^{2}} n x^{2}+\frac{n^{2 \mid m}}{2.1^{4 \mid 1} m^{3}} n x^{3} \\
& +\frac{15 n^{3}+30 n^{2} m+5 n m^{2}-2 m^{3}}{48.1^{5 \mid 1} m^{4}} n x^{4} \\
& +\frac{3 n^{4}+10 n^{3} m+5 n^{2} m-2 n m}{16.1^{6 \mid !} m^{5}} n x^{5} \\
& +\ldots . \cdots) \\
\text { 7) } \boldsymbol{p}-\frac{n}{m}=\frac{1}{x^{\frac{n}{m}}}\left(1-\frac{n x}{2 m}\right. & +\frac{3 n-m}{1^{4 \mid !} m^{2}} n x^{2}-\frac{n^{2 \mid-m}}{2.1^{4 \mid 1} m^{3}} n x^{3} \\
& +\frac{15 n^{3}-30 n^{2} m+55 n m^{2}+2 m^{3}}{48.1^{5 \mid 1} m^{4}} n x^{4} \\
& +\frac{3 n^{4}-10 n^{3} m+5 n^{2} m^{2}+2 n m^{3}}{10.1^{6 \mid 1} m^{5}} n x^{5}
\end{aligned}
$$

§. 18.

Es lässt sich auch die $n$ te Potenz des Polynomiums

$$
\text { 1) } Q=1+1+\frac{x}{1}+\frac{x^{2}}{1.2}+\frac{x^{3}}{1.2 .3}+\ldots=1+e^{x}
$$

darstellen. Es ist aus (1. \$. 17.)

$$
\text { 2) } Q^{n}=\left(2+\left(e^{x}-1\right)\right)^{n}=(2+P)^{n}
$$

also auch

$$
\text { 3) } Q^{n}=2^{n}+n \cdot 2^{n-1} p^{1}+(n)_{2} 2^{n-2} P^{2}+(n)_{3} P^{3}+\ldots
$$

Für diese Darstellung sind nur die positiven Potenzen von $\boldsymbol{P}$ nöthig. Benutzt man die Gleichung (1. \$. 17.) und setzt dort der Reihe nach 1, 2, $3, \ldots$ statt $n$, so ergiebt sich: 
4) $Q^{n}=2^{n}+n \cdot 2^{n-1}\left(x+\frac{S C^{\prime}(1)^{1}}{2} x^{2}+\frac{S C^{\prime}(1)^{2}}{2.2} x^{2}+\frac{S C^{\prime}(1)^{3}}{2.3 .4} x^{3}+\ldots\right)$

$$
\begin{aligned}
& +(n)_{2} 2^{n-2}\left(x^{2}+\frac{S C^{\prime}(1,2)^{1}}{3} x^{3}+\frac{S C^{\prime}(1,2)^{2}}{3.4} x^{4}+\frac{S C^{\prime}(1,2)^{3}}{3.4 .5} x^{4}+\ldots\right) \\
& +(n)_{3} 2^{n-3}\left(x^{3}+\frac{S C^{\prime}(1,2,3)^{1}}{4} x^{4}+\frac{S C^{\prime}(1,2,3)^{2}}{4.5} x^{5}+\frac{S C^{\prime}(1,2,3)^{3}}{4.5 .6} x^{6}+\ldots .\right)
\end{aligned}
$$

Wird dieser Ausdruck nach den Potenzen von $x$ geordnet, so ergiebt sich:

5) $Q^{n}=2^{n}+n \cdot 2^{n-1} x+\left(n .2^{n-1} S C^{\prime}(1)^{1}+n^{2^{2-1}} \cdot 2^{n-2}\right) \frac{x^{2}}{1^{2 \mid 1}}$

$$
\begin{gathered}
+\left[n .2^{n-1} S C^{\prime}(1)^{2}+n^{2 \mid-1} 2^{n-2} S C^{\prime}(1,2)^{1}+n^{3 \mid-1} 2^{n-3}\right] \frac{x^{3}}{1^{2 \mid 1}} \\
+\left[n .2^{n-1} S C^{\prime}(1)+n^{2 \mid-1} 2^{n-2} S C^{\prime}(1,2)^{2}+n^{3 \mid-1} 2^{n-3} S C^{\prime}(1,2,3)^{1}+n^{4 \mid-1} \cdot 2^{n-4}\right] \frac{x^{4}}{1^{4 \mid 1}}
\end{gathered}
$$

Die Vorzahl des $(r+1)$ ten Gliedes hat folgende Gestalt:

6) $A_{r}=n \cdot 2^{n-1} \frac{S C^{\prime}(1)^{r-1}}{2^{r-1 \mid 1}}+(n)_{2} \cdot 2^{n-2} \frac{S C^{\prime}(1,2)^{r-2}}{3^{r-2 \mid 1}}+(n)_{3} \cdot 2^{n-3} \frac{S C^{\prime}(1,2,3)^{r-3}}{4^{r-3 \mid 1}}+\ldots$

$$
\ldots+(n)_{r} \cdot 2^{n-r} \frac{S C^{u}(1,2, \ldots . r)^{0}}{(r+1)^{0}} \text {. }
$$

Scheidet man die Facultät, welche in dem Nenner der Glieder vorkommt, aus, und führt die Zahlenwerthe für die vorstehenden Symbole nach (\$.9., 8. und 25.) ein, so bekommt das $(r+1)$ te Glied folgende Form:

$$
\begin{aligned}
& \text { 7) } A_{r} . x^{n}=\left[n .2^{n-1}+n^{2 \mid-1} 2^{n-2}\left(2^{r-1}-1\right)\right. \\
& +\frac{n^{3 \mid-1} \cdot 2^{n-3}}{1^{2 \mid 1}}\left(3^{r-1}-2.2^{r-1}+1\right) \\
& +\frac{n^{4 \mid-1} \cdot 2^{n-4}}{1^{3 \mid 1}}\left(4^{r-1}-3.3^{r-1}+3.2^{n-1}-1\right) \\
& +n^{r-3 \mid-1} 2^{n-r+3} \cdot \frac{(r-3)^{2 \mid 1}}{1^{2 \mid 1}} \cdot \frac{r^{4 \mid-1}}{1^{4 \mid 1}} \\
& +n^{r-2 \mid-1} 2^{n-r+2} \cdot \frac{3(r-2)+1}{4} \cdot \frac{(r-2)^{3 / 1}}{1^{3 / 1}} \\
& +n^{r-1 \mid-1} 2^{n-r+1} \cdot \frac{(r-1)^{2 \mid 1}}{1^{2 \mid 1}} \\
& \left.+n^{r \mid-1} 2^{n-r}\right] \frac{x^{r}}{1^{r \mid 1}} \text {. }
\end{aligned}
$$

Die Gleichung (6.) gilt für ein positives und negatives, ganzes und gebrochenes $n$. Sie giebt für unsern $Z_{\text {weck }}$ folgende Ausdrücke: 
8) $\left(1+e^{x}\right)^{n}=2^{n}+n \cdot 2^{n-1} x+\left(n \cdot 2^{n-1}+n^{2 \mid-1} 2^{n-2}\right) \frac{x^{2}}{1^{2 \mid 1}}$

$$
\begin{aligned}
& +\left(n \cdot 2^{n-1}+n^{2 \mid-1} \cdot 2^{n-2} \cdot 3+n^{3 \mid-1} 2^{n-3}\right) \frac{x^{3}}{1^{3 \mid 1}} \\
& +\left(n \cdot 2^{n-1}+n^{2 \mid-1} \cdot 2^{n-2} \cdot 7+n^{3 \mid-1} \cdot 2^{n-3} \cdot 6+n^{4 \mid-1} \cdot 2^{n-4}\right) \frac{x^{4}}{1^{4 \mid 1}}
\end{aligned}
$$

$$
+\left(n \cdot 2^{n-1}+15 \cdot n^{2 \mid-1} \cdot 2^{n-2}+25 \cdot n^{3 \mid-1} \cdot 2^{n-3} \cdot+10 n^{4 \mid-1} \cdot 2^{n-4}+n^{5 \mid-1} 2^{n-5}\right) \frac{x^{5}}{1^{5,1}}
$$

9)

$$
\begin{aligned}
\frac{1}{\left(1+e^{x}\right)^{n}}=\frac{1}{2^{n}}-\frac{n x}{2^{n+1}} & +\frac{1}{2^{n}}\left(-\frac{n}{2}+\frac{n^{2 / 1}}{2^{2}}\right) \frac{x^{2}}{1^{5,1}} \\
& +\frac{1}{2^{n}}\left(\frac{-n}{2}+\frac{3 n^{211}}{2^{2}}-\frac{n^{31}}{2^{3}}\right) \frac{x^{3}}{1^{3 ! 1}} \\
& +\frac{1}{2^{n}}\left(\frac{-n}{2}+\frac{7 n^{2 / 1}}{2^{2}}-\frac{6 n^{31}}{2^{3}}+\frac{n^{4: 1}}{2^{4}}\right) \frac{x^{4}}{1^{411}}
\end{aligned}
$$

10) $\left(1+e^{x}\right)^{\frac{n}{m}}=2^{\frac{n}{m}}\left[1+\frac{n}{m \cdot 2} x+\left(\frac{n}{m \cdot 2}+\frac{n^{2 \mid-m}}{2^{2} m^{2}}\right) \frac{x^{2}}{1^{211}}\right.$

$$
\begin{aligned}
& +\left(\frac{n}{2 m}+\frac{3 n^{2 \mid-m}}{2^{2} \cdot m^{2}}+\frac{n^{3 \mid-m}}{2^{3} m^{3}}\right) \frac{x^{3}}{1^{311}} \\
& +\left(\frac{n}{2 m}+\frac{7 n^{2 \mid-m}}{2^{2} m^{2}}+\frac{6 n^{3 \mid-m}}{2^{3} m^{3}}+\frac{n^{4 \mid-m}}{2^{4} m^{4}}\right) \frac{x^{4}}{1^{4,1}}
\end{aligned}
$$

11)

$$
\begin{aligned}
\left(1+e^{x}\right)^{-\frac{n}{m}}=\frac{1}{2^{\frac{n}{m}}}\left[1-\frac{n x}{2 m}\right. & +\left(-\frac{n}{2 m}+\frac{n^{2 \mid n}}{2^{2} m^{2}}\right) \frac{x^{2}}{1^{2 \mid 1}} \\
& +\left(-\frac{n}{2 m}+\frac{3 n^{2 \mid m}}{2^{2} m^{2}}-\frac{n^{3 \mid m}}{2^{3} m^{3}}\right) \frac{x^{3}}{1^{311}} \\
& +\left(-\frac{n}{2 m}+\frac{7 \cdot n^{2 \mid m}}{2^{2} m^{2}}-\frac{6 n^{3 \mid m}}{2^{3} m^{3}}+\frac{n^{4 \mid m}}{2^{4} m^{4}}\right) \frac{x^{4}}{1^{411}}
\end{aligned}
$$

Vergleicht man die Ausdrücke in (\$. 16. bis 18.) mit denen, welche die Analysis bisher gab, so wird man die Vortheile wahrnehmen, welche die in (\$. 8. und 9.) gefundenen Resultate gewähren. Die Ausdrücke lösen nämlich auf eine eben so einfache als allgemeine Weise Probleme auf, welche durch lange Abhandlungen noch nicht auf befriedigende Weise gelöset waren. Die Resultate, welche bisher gefunden wurden, standen unter sich isolirt und erstreckten sich entweder nur auf ein positives, oder auf ein negatives $n$. Dabei wurde gewöhnlich nur eine zurücklaufende Bildungsart ermittelt. Ausdrücke für gebrochene Exponenten waren ausgeschlossen. Entwickelte Ausdrücke, welche die Erhebung der genannten Polynomien in Potenzen bisher gaben, 
sind mir nicht bekannt. Deswegen habe ich sie hier mitgetheilt. Wäre auch mit den in ( $\$$. 16. bis 18.) gefundenen Resultaten der Umfang der Anwendbarkeit der in ( $\$$. 8. und 9.) gefundenen Gleichungen geschlossen, so würde auch dies schon genügen, um die Wichtigkeit jener Gleichungen hervorzuheben. Dies ist jedoch nicht der Fall. Ihre Anwendbarkeit findet auch noch in der Differenzenrechnung und bei den Functionen Statt, die ich mit dem Namen Aufstufungen bezeichnet habe; wie im Folgenden gezeigt werden soll.

\section{§. 19.}

Bekanntlich lässt sich der Unterschied einer Function durch Differentıale dieser Function darstellen. Bei dieser Darstellung sind die Summenausdrücke der Verbindungen mit Wiederholungen nöthig (S. 12ter Bd. d. Journ. S. 333.). Die Gleichung, auf welche die Differenzenrechnung führt, s:t

$$
\text { 1) } \begin{gathered}
\Delta^{n} x=\frac{\partial^{n} x(\Delta x)^{n}}{(\partial x)^{n}}+\frac{S C^{\prime}(1,2, \ldots n)^{1}}{n+1} \frac{\partial^{n+1} x}{(\partial x)^{n+1}}(\Delta x)^{n+1}+\frac{S C^{\prime}(1,2, \ldots . n)^{2}}{(n+1)^{2 \mid 1}} \frac{\partial^{n+2} x}{(\partial x)^{n+2}}(\Delta x)^{n+2}+\ldots . \\
\ldots .+\frac{S C^{\prime}(1,2, \ldots . n)^{r}}{(n+1)^{r+1}} \cdot \frac{\partial^{n+r} x}{(\partial x)^{n+r}}(\Delta x)^{n+r}+\ldots
\end{gathered}
$$

Unter $X$ wird jede beliebige Function von $x$ verstanden. Dieser Ausdruck gilt also für die Entwicklung der Unterschiede der Functionen überhaupt. Werden nun die angezeigten Werthe aus (\$. 9.) in (1.) eingeführt, so erhält man ganz allgemein:

$$
\text { 2) } \begin{aligned}
\Delta^{n} X=\frac{\partial^{n+1} X}{(\partial x)^{n+1}}(\Delta x)^{n} & +\frac{n}{2} \frac{\partial^{n+1} X}{(\partial x)^{n+1}}(\Delta x)^{n+1}+\frac{3 n+1}{1^{411}} n \cdot \frac{\partial^{n+2} X}{(\partial x)^{n+2}}(\Delta x)^{n+2} \\
& +\frac{n^{211} \cdot n}{2 \cdot 1^{411}} \frac{\partial^{n+3} X}{(\partial x)^{n+3}}(\Delta x)^{n+3} \\
& +\frac{15 n^{3}+30 n^{2}+5 n-2}{48.1^{1 / 1}} n \cdot \frac{\partial^{n+4} X}{(\partial x)^{n+4}}(\Delta x)^{n+4} \\
& +\frac{3 n^{4}+10 n^{3}+5 n-2 n}{16 \cdot 1^{6 \cdot 1}} n \cdot \frac{\partial^{n+5} X}{\partial x)^{n+5}}(\Delta x)^{n+5} \\
& +\frac{63 n^{5}+315 n^{4}+315 n^{3}-91 n-42 n+16}{144.1^{11}} n \cdot \frac{\partial^{n+6} X}{(\partial x)^{n+5}}(\Delta x)^{n+6}
\end{aligned}
$$

Diese Reihe bricht ab, wenn eines der hühern Differentiale in 0 übergeht. Der Ausdruck ist, wie gesagt, ganz allgemein; er gilt nicht nur für ein positives, sondern auch für ein negatives $n$, und kann sogar auf ein gebrochenes $n$ ausgedehnt werden, wenn man dafür eine Bedeutung findet. Es ist zunächst 
3) $\Delta^{-n} X=\frac{\partial^{-n} X}{(\partial x)^{-n}}(\Delta x)^{-n}-\frac{n}{2} \cdot \frac{\partial^{-n+1} X}{(\partial x)^{-n+1}}(\Delta x)^{-n+1}+\frac{3 n-1}{1^{4 / 1}} n \frac{\partial^{-n+2} X}{(\partial x)^{-n+2}}(\Delta x)^{-n+2}$

$$
\begin{aligned}
& -\frac{n^{2 \mid-1}}{2.1^{4 / 1}} \cdot n \cdot \frac{\partial^{-n+3} X}{(\partial x)^{-n+3}}(\Delta x)^{-n+3} \\
& +\frac{15 n^{3}-30 n^{2}+5 n+2}{48.1^{5 / 1}} n \frac{\partial^{-n+4} X}{(\partial x)^{-n+4}}(\Delta x)^{-n+4}
\end{aligned}
$$

Erwägt man, dass negative Differentiale den positiven gegenüber in demselben Verhältnisse stehen, wie die positiven Unterschiede den negativen gegenüber, und dass sie also Integrale bedeuten, so lässt sich aus (3.) auch folgende Formel ableiten:

$$
\begin{aligned}
& \text { 4) } \Delta^{-n} X=\frac{1}{(\Delta x)^{n}} \int^{n} X(\partial x)^{n}-\frac{n}{2(\Delta x)^{n-1}} \int^{n-1} X(\partial x)^{n-1}+\frac{(3 n-1) n}{1^{411}(\Delta x)^{n-2}} \int^{n-2} X(\partial x)^{n-2} \\
& -\frac{n^{2 \mid-1} n}{2.1^{111}(\Delta x)^{n-3}} \int^{n-3} X(\partial x)^{n-3} \\
& +\frac{15 n^{3}-30 n^{2}+5 n+2}{48.1^{111}(\Delta x)^{n-4}} n \int^{n-4} X(\partial x)^{n-4}
\end{aligned}
$$

Hier ist $\boldsymbol{f}^{0}=1$ und die Integrale gehen wieder in Differentiale über, wenn der Exponent des Integralzeichens negativ wird. Man kann nun in die Gleichungen (2. bis 5.) statt $n$ sogar eine gebrochene Zahl setzen und erhält dann folgende Ausdrïcke:

5) $\Delta^{\frac{n}{m}} X=\frac{\partial^{\frac{n}{m}} \mathbf{X}}{(\partial x)^{\frac{n}{m}}}(\Delta x)^{\frac{n}{m}}+\frac{n}{2 m} \frac{\partial^{\frac{n}{m}+1} X}{(\partial x)^{\frac{n}{m}+1}}(\Delta x)^{\frac{n}{m}+1}+\frac{2 n+m}{1^{41} m^{2}} n \cdot \frac{\partial^{\frac{n}{m}+2} X}{(\partial x)^{\frac{n}{m}+2}}(\Delta x)^{\frac{n}{m}+1}$

$$
\begin{aligned}
& +\frac{n^{2 m} \cdot m}{1^{211} \cdot 1^{411} \cdot m^{3}} \frac{\partial^{\frac{n}{m}+3} X}{(\partial x)^{\frac{n}{m}+3}}(\Delta x)^{\frac{n}{m}+3} \\
& +\frac{15 n^{3}+30 n^{2} m+5 n m^{2}-2 m^{3}}{48.1^{511} m^{4}} n \cdot \frac{\partial^{\frac{n}{m}+4} X}{(\partial x)^{\frac{n}{m}+4}}(\Delta x)^{\frac{n}{m}}
\end{aligned}
$$

6) $\Delta^{-\frac{n}{m}} X=\frac{\partial^{-\frac{n}{m}} X}{(\partial x)^{-\frac{n}{m}}(\Delta x)^{\frac{n}{m}}}-\frac{n}{2 m} \cdot \frac{\partial^{-\frac{n}{m}+1} X}{(\partial x)^{-\frac{n}{m}+1}(\Delta x)^{\frac{n}{m}-1}}+\frac{3 n-m}{1^{411} m^{2}} n \cdot \frac{\partial^{-\frac{n}{m}+2} X}{(\partial x)^{-\frac{n}{m}+3}(\Delta x)^{\frac{n}{m}-2}}$

$$
\begin{aligned}
& -\frac{n^{2 \mid-m}}{2.1^{411} m^{3}} \cdot n \frac{\partial^{-\frac{n}{m}+3} X}{(\partial x)^{-\frac{n}{m}+3}(\Delta x)^{\frac{n}{m}-3}} \\
& +\frac{15 n^{3}-30 n^{2} m+5 n m^{2}+2 m^{3}}{48.1^{5 / 1} m^{4}} n \frac{\partial^{-\frac{n}{m}+4} X}{(\partial x)^{-\frac{n}{m}+4} \Delta^{\frac{n}{m}-4}}
\end{aligned}
$$


Hierdurch sind formell die Werthe für $\Delta^{\frac{n}{m}} X$ und $\Delta^{-\frac{n}{m}} X$ dargestellt. Was aber die Bedeutung des Unterschiedes einer Function mit gebrochenen, positiven oder negativen Exponenten sei, geht daraus nicht hervor, während doch die entwickelten Darstellungen der Unterschiede mit gebrochenen Exponenten möglich sind. Diese Darstellungen werden immer dann möglich sein, wenn $\partial^{\frac{n}{m}} X$ und $\partial^{-\frac{n}{m}} X$ möglich ist. Durch Aufsuchen eines allgemeinen Gesetzes, welches die vorstehenden Ausdrücke in sich fasst, wird die Aufgabe gelöset werden, und es entsteht die Frage, welche Bedeutung und Anwendung die auf dem angegebenenem Wege gefundenen Resultate erhalten werden, oder können?

Wenden wir die in ( 1 bis 6 ) gegebenen Formeln, welche für alle Functionen gelten, auf einen besondern Fall an und wählen wir dazu $X=x^{p}$, so ergiebt sich aus (2.), da $\frac{\partial^{r} x^{p}}{(\partial x)^{r}}=p^{r \mid-1} x^{p-1}$ ist, folgender entwickelte Ausdruck für den $n$ ten Unterschied einer Potentialfunction:

7)

$$
\begin{aligned}
\Delta^{n} x^{p}=p^{n !-1} x^{p-n}(\Delta x)^{n} & +\frac{n}{1} \cdot p^{n+11-1} x^{p-n-1}(\Delta x)^{n+1} \\
& +\frac{3 n+1}{1^{411}} n \cdot p^{n+21-1} x^{p-n-2}(\Delta x)^{n+2} \\
& +\frac{n^{211} n}{2.1^{41}} p^{n+31-1} x^{p-n-3}(\Delta x)^{n+3} \\
& +\frac{15 n^{3}+30 n^{2}+5 n-2}{48.1^{51}} n p^{n-4 \mid-1} x^{p-n-4}(\Delta x)^{n+1}
\end{aligned}
$$

Da dieser Ausdruck für ein positives und negatives, ganzes und gebrochenes $n$ gilt, so ergiebt sich daraus

8) $\Delta^{-n} x^{p}=\frac{p^{-n \mid-1} x^{p+n}}{(\Delta x)^{n}}-\frac{n}{2} \cdot \frac{p^{-n+1 \mid-1} x^{p+n-1}}{(\Delta x)^{n-1}}+\frac{3 n-1}{1^{411}} n \cdot \frac{p^{-n+21-1} x^{p+n-2}}{(\Delta x)^{n-2}}$

$$
\begin{aligned}
& -\frac{n^{21-1} \cdot n}{2.1^{411}} \cdot \frac{p^{-n+31-1} x^{p+n-3}}{(\Delta x)^{n-3}} \\
& +\frac{15 n^{3}-30 n^{2}+5 n+2}{48.1^{511}} n \cdot \frac{p^{-n+4 \mid-1} x^{p+n-1}}{(\Delta x)^{n-4}}
\end{aligned}
$$

Für ein gebrochenes $n$ erhält man : 
9) $\Delta^{\frac{n}{m}} x^{\rho}=\left(\frac{\Delta x}{x}\right)^{\frac{n}{m}}\left[p^{\frac{n}{m} \mid-1} x^{\rho}+\frac{n}{2 m} \cdot p^{\frac{n}{m}+1 \mid-1} x^{\nu-1} \Delta x\right.$

$$
\begin{aligned}
& +\frac{3 n+m}{1^{411} m^{2}} n \cdot p^{\frac{n}{m}+2 \mid-1} x^{p-2}(\Delta x)^{2} \\
& +\frac{n^{2 \mid m} \cdot n}{2.1^{41} m^{3}} \cdot p^{\frac{n}{m}+31-1} x^{p-3}(\Delta x)^{3}
\end{aligned}
$$

10) $\Delta^{-\frac{n}{m}} x^{p}=\left(\frac{x}{\Delta x}\right)^{\frac{n}{m}}\left[\frac{x^{p}}{\left(p+\frac{n}{m}\right)^{\frac{n}{m} \mid-1}}-\frac{n \cdot p^{-\frac{n}{m}+11-1}}{2 . m} x^{p-1} \Delta x\right.$

$$
\begin{aligned}
& +\frac{3 n-m}{1^{411} m^{2}} \cdot n \cdot p^{-\frac{n}{m}+2 \mid-1} x^{p-2}(\Delta x)^{2} \\
& -\frac{n^{21-m} \cdot n}{2.2^{411}} n \cdot p^{\frac{n}{m}+3 \mid-1} x^{p-3}(\Delta x)^{3}
\end{aligned}
$$

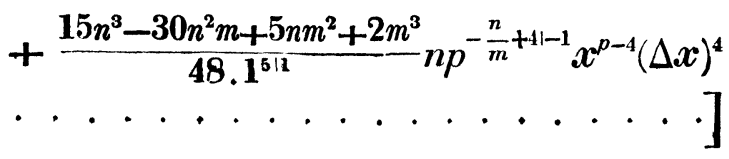

Alle in (9. und 10.) angezeigten Operationen sind ausführbar; also wird sich auch der Werth von $\Delta^{\frac{n}{m}} x^{p}$ und $\Delta^{-\frac{n}{m}} x^{p}$ darstellen lassen, und es kann sich daher, wie bemerkt, nur um seine Bedeutung und Anwendung handeln. Der weitere Erfolg unserer Untersuchungen wird Gelegenheit zur Anwendung geben.

Die Formel (8.) lässt sich auch so geben:

$$
\text { 11) } \begin{aligned}
\Delta^{-n} x^{p}=\frac{p^{-n \mid-1} x^{p+n}}{(\Delta x)^{n}} & -\frac{S C(1,2, \ldots . n-1)^{1}}{n-1} \cdot \frac{p^{-n+11-1} x^{p+n-1}}{(\Delta x)^{n-1}} \\
& +\frac{S C(1,2, \ldots n-1)^{2}}{(n-1)^{2 \mid-1}} \cdot \frac{p^{-n+2 \mid-1} x^{p+n-2}}{(\Delta x)^{n-2}} \\
& -\frac{S C(1,2, \ldots n-1)^{3}}{(n-1)^{3 \mid-1}} \cdot \frac{p^{-n+3 \mid-1} x^{p+n-3}}{(\Delta x)^{n-3}}
\end{aligned}
$$

Ein specieller Fall der Gleichung (4., 8. oder 11.), wenn nämlich $n=1$ gesetzt wird, ist bekannt. In diesem Falle entstehen die Bernoullischen Zahlen. Nennt man der Reibe nach diese Zahlen (auch diejenigen nicht ausgeschlossen, welche auf 0 führen) $A_{1}, A_{2}, A_{3} \ldots$, so erhält man:

12) $\frac{S C(1,2, \ldots . n-1)^{1}}{n-1}=A_{1}, \frac{S C(1,2, \ldots n-1)^{2}}{(n-1)^{2 \mid-1}}=A_{2}, \frac{S C(1,2, \ldots n-1)^{3}}{(n-1)^{3-1}}=A_{3} \ldots$

$$
\ldots \frac{S C(1,2, \ldots . n-1)^{r}}{(n-1)^{r \mid-1}}=A_{r},
$$

wenn in der entwickelten Darstellung von $\frac{S C(1,2, \ldots . n-1)^{r}}{(n-1)^{r 1-1}} n=1$ gesetzt wird. 
Hiezu dienen die Ausdrücke (9., 10., 16. §. 9.) und es zeigt sich, dass dic Bernoullischen Zahlen nur ein besonderer Fall der Summenausdrücke sind, welche von den Verbindungen ohne Wiederholungen zu der 1ten, 2ten, 3ten, 4ten .... Classe gelten; wie es die Gleichung (12.) zeigt.

Der eben berührte Fall, wenn in (4.) $n=1$ gesetzt wird, soll hier noch hervorgehoben werden, da er später zu weitern Anwendungen dienen wird. Es ist

$$
\begin{aligned}
\Delta^{-1} X=\frac{1}{\Delta x} \int X \partial x & +{ }_{2}^{1} X+\frac{1}{12} \frac{\partial X}{\partial x} \cdot \Delta x \\
& -\frac{1}{120} \cdot \frac{\partial^{3} X}{1.2 .3(\partial x)^{3}}(\Delta x)^{3} \\
& +\frac{1}{252} \cdot \frac{\partial^{5} X}{1.2 \ldots 5(\partial x)^{5}}(\Delta x)^{5} \\
& -\frac{1}{240} \cdot \frac{\partial^{7} X}{1.2 \ldots . .7(\partial x)^{7}}(\Delta x)^{7}
\end{aligned}
$$

§. 20.

Ausser den Anwendungen im vorigen Paragraph ergiebt sich noch eine andere für die Darstellung der Aufstufung einer Function durch ibre Differentiale. In der Differenzen-Rechnung (11ter Bd. d. Journ. S. 185. §. 17. und 20.) ist gezeigt worden, dass folgende Gleichung gilt:

$$
\text { 1) } \zeta^{ \pm n} X=\left(1+e^{\frac{\Delta x . \partial}{\partial x}}\right)^{ \pm n} X
$$

wenn $X$ eine Function von $x$ ist. Nun ist hier oben in ( $§ .18$.$) die Ent-$ wicklung des Binomiums

$$
Q^{n}=\left(1+e^{x}\right)^{n}
$$

für ein positives und negatives, ganzes und gebrochenes $n$ gegeben. Werden die entsprechenden Werthe in die dortigen Resultate eingeführt, so ergiebt sich unmittelbar für die positive und negative Aufstufung einer Function durch ihre Differentiale Folgendes: 
2) $\quad \zeta^{n} X=2^{n} X+n \cdot 2^{n-1} \frac{\partial X}{\partial x} \Delta x$

$$
\begin{aligned}
& +\left(n \cdot 2^{n-1}+n^{2 \mid-1} \cdot 2^{n-2}\right) \frac{\partial^{2} X}{1.2(\partial x)^{2}}(\Delta x)^{2} \\
& +\left(n \cdot 2^{n-1}+3 n^{2 \mid-1} \cdot 2^{n-2}+2^{n-3} \cdot n^{3 \mid-1} 2^{n-3}\right) \frac{\partial^{3} X}{1^{3 \mid 1}(\partial x)^{3}}(\Delta x)^{3} \\
& +\left(n \cdot 2^{n-1}+7 \cdot n^{2 \mid-1} \cdot 2^{n-2}+6 n^{3 \mid-1} 2^{u-3}+n^{4 \mid-1} 2^{n-4}\right) \frac{\partial^{4} X}{1^{4 \mid 1}(\partial x)^{4}}(\Delta x)^{4}
\end{aligned}
$$

3) $\zeta^{-n} X=\frac{X}{2^{n}}-\frac{n}{2^{n+1}} \cdot \frac{\partial X}{\partial x} \Delta x$

$$
\begin{aligned}
& +\frac{1}{2^{n}}\left(-\frac{n}{2}+\frac{n^{211}}{2^{2}}\right) \frac{\partial^{2} X}{1.2(\partial x)^{2}}(\Delta x)^{2} \\
& +\frac{1}{2^{n}}\left(-\frac{n}{2}+3 \cdot \frac{n^{211}}{2^{2}}-\frac{n^{311}}{2^{3}}\right) \frac{\partial^{3} X}{1^{311}(\partial x)^{3}}(\Delta x)^{3} \\
& +\frac{1}{2^{n}}\left(-\frac{n}{2}+\frac{7 \cdot n^{211}}{2^{2}}-\frac{6 \cdot n^{311}}{2^{3}}+\frac{n^{41}}{2^{4}}\right) \frac{\partial^{4} X}{1^{411}(\partial x)^{4}}(\Delta x)^{4}
\end{aligned}
$$

4) $\quad^{\frac{n}{m}} X=2^{\frac{n}{m}}\left(X+\frac{n}{2 m} \cdot \frac{\partial X}{\partial x} \Delta x\right.$

$$
\begin{aligned}
& +\left(\frac{n}{2 m}+\frac{n^{21-m}}{2^{2} m^{2}}\right) \frac{\partial^{2} X}{1.2(\partial x)^{2}}(\Delta x)^{2} \\
& +\left(\frac{n}{2 m}+\frac{3 \cdot n^{2 \mid-m}}{2^{2} m^{2}}+\frac{n^{2 \mid-m}}{2^{3} m^{3}}\right) \frac{\partial^{3} X}{1^{311}(\partial x)^{3}}(\Delta x)^{3}
\end{aligned}
$$

5) $\zeta^{-\frac{n}{m}} X=\frac{1}{2^{\frac{n}{m}}}\left[X-\frac{n}{2 m} \frac{\partial X}{(\partial x)^{2}} \Delta x\right.$

$$
\begin{aligned}
& +\left(\frac{-n}{2 m}+\frac{n^{2 \mid m}}{2^{2} m^{2}}\right) \frac{\partial^{2} X}{1.2(\partial x)^{2}}(\Delta x)^{2} \\
& +\left(\frac{-n}{2 m}+\frac{3 n^{2 \mid m}}{2^{2} m^{2}}-\frac{n^{3 / m}}{2^{3} m^{3}}\right) \frac{\partial^{3} X}{{1^{311}}^{2}(\partial x)^{3}}(\Delta x)^{3}
\end{aligned}
$$

Ist $n=1$, so ergiebt sich aus (3.):

$$
\text { 6) } \quad \begin{aligned}
\zeta^{-1} X=\frac{x}{2}-\frac{\partial X}{4 \partial x} \Delta & x+\frac{1}{8} \cdot \frac{\partial^{3} X}{1.2 .3(\partial x)^{3}}(\Delta x)^{3}-\frac{1}{4} \frac{\partial^{5} X \cdot(\Delta x)^{5}}{1.2 .3 .4 .5(\partial x)^{5}} \\
& +\frac{17}{16} \frac{\partial^{7} X}{1^{71}(\partial x)^{7}}(\Delta x)^{7} \ldots
\end{aligned}
$$


Hieraus folgt das allgemeine Gesetz für die positiven und negativen Aufstufungen der Potentialfunctionen mittels der Gleichung

$$
\frac{\partial^{r} x^{p}}{(\partial x)^{r}}=p^{r \mid-1} x^{\rho-r}
$$

unmittelbar. Es ist nämlich

6) $\zeta^{n} x^{p}=2^{n} x^{p}+n \cdot 2^{n-1} p x^{p-1} \Delta x$

$$
\begin{aligned}
& +\left(n \cdot 2^{n-1}+n^{2 \mid-1} 2^{n-2}\right)(p)_{2} x^{p-2}(\Delta x)^{2} \\
& +\left(n \cdot 2^{n-1}+3 n^{2 \mid-1} 2^{n-2}+n^{3 \mid-1} 2^{n-3}\right)(p)_{3} x^{p-3}(\Delta x)^{3} \\
& +\left(n \cdot 2^{n-1}+7 . n^{21-1} 2^{n-2}+6 n^{3 \mid-1} \cdot 2^{n-3}+n^{4-1} \cdot 2^{n-4}(p)_{4} x^{p-4}(\Delta x)^{4}\right.
\end{aligned}
$$

7) $\zeta^{-n} x^{p}=\frac{x^{p}}{2^{n}}-\frac{n \cdot x^{p-1}}{2^{n+1}} \Delta x+\frac{1}{2^{n}}\left(-\frac{n}{2}+\frac{n^{211}}{2^{2}}\right)(p)_{2} x^{p-2}(\Delta x)^{2}$

$$
\begin{aligned}
& +\frac{1}{2^{n}}\left(-\frac{n}{2}+3 \frac{n^{2 / 1}}{2^{2}}-\frac{n^{311}}{2^{3}}\right)(p)_{3} x^{p-3}(\Delta x)^{3} \\
& +\frac{1}{2^{n}}\left(-\frac{n}{2}+\frac{7 n^{211}}{2^{2}}-\frac{6 n^{311}}{2^{3}}+\frac{n^{411}}{2^{4}}\right)(p)_{4} x^{\mu-4}(\Delta x)
\end{aligned}
$$

Es ist jetzt nicht schwierig, die Aufstufungen für gebrochene Exponenten zu finden. Specielle Fälle ergeben sich aus den obigen Darstellungen leicht. Der Fall, wenn in (7.) $n=1$ gesetzt wird, ist zu bemerken. Es findet sich für die erste negative Abstufung von $x^{p}$ :

8)

$$
\begin{aligned}
\zeta^{-1} x^{p}=\frac{x^{p}}{2} & -\frac{1}{4} p x^{p-1} \Delta x \\
& +\frac{1}{8} \cdot \frac{p(p-1)(p-2)}{1.2 .3} x^{p-3}(\Delta x)^{3} \\
& -\frac{1}{4} \cdot \frac{p(p-1) \ldots(p-4)}{1.2 \ldots 5} x^{p-5}(\Delta x)^{5} \\
& +\frac{17}{16} \frac{p(p-1) \ldots(p-6)}{1.2 \ldots .6} x^{p-6}(\Delta x)^{\tau}
\end{aligned}
$$

Die Vorzahlen der Glieder dieser Reihe stehen den Bernoullischen Zahlen zur Seite, und die vorstehende Reihe hat bei Summirung der Potenzenreihen, deren Glieder abxechselnde Zeichen haben, dieselbe Bedeutung, wie die Reihe von Bernoulli für die Summirung der Potenzenreihen, deren Glieder einerlei Zeichen haben. Die in diesem und dem vorhergehenden 
Paragraph gefundenen Resultate sind für die Rechnung mit Differenzen wichtig. Die Entwicklungen von $\Delta^{n} X$ und $\Delta^{-n} X$, so wie von $\zeta^{n} X$ und $\zeta^{-n} X$ wurden sonst immer getrennt behandelt. Es waren daher eine Menge von Operationen zur Darstellung der isolirten Fälle nöthig, die hier zusammen ihre Erledigung finden. Wenn auch Methoden angegeben wurden, wie man zu diesen Entwicklungen gelangen könne, so fehlten doch immer die entwickelten Ausdrücke, die oben mitgetheilt sind. Hievon kann man sich überzeugen, wenn man die hierher gehörigen Schriften, u. a. Traité d. calc. diff. et intég. p. Lacroix T. III. etc. nachsieht. Das allgemeine Gesetz der Aufstufungen, welches ich schon früher (im 1lten Bd. d. Journ.) zu finden mich bemühte, ergiebt sich hier und bringt, wie bei den Unterschieden, alle nöthigen Entwicklungen auf eine allgemeine und einfache Basis zurück. 


\section{IV.}

§. 21.

Die in (19. und 20.) aufgestellten Gleichungen geben ein Mittel an die Hand, den natürlichen Logarithmen einer Facultät auszudrücken. Es ist

1) $\lg x^{n+1 \mid d}=\lg x+\lg (x+d)+\lg (x+2 d) \ldots \lg (x+n d)$.

Nach der Differenzenrechnung (S. d. Journ. 14ter Bd. S. 262 u. ff.) ist

2) $X_{0}+X_{1}+X_{2}+\ldots .+X_{n}=\Delta^{-1} X_{n+1}-\Delta^{-1} X$.

Setzt man nun $X_{0}=\lg x, X_{1}=\lg (x+d), X_{2}=\lg (x+2 d)$ u. s. w. $X^{n}=\lg (x+n d)$, so erhält man, mit Rücksicht auf (1. u. 2. und §. 19., 12.):

$$
\begin{aligned}
\lg x^{n+1 \mid d}= & \Delta^{-1} \lg (x+(n+1) d)-\Delta^{-1} \lg x \\
= & \int \frac{\lg (x+(n+1) d)}{d} d x-\int \frac{\lg x}{d} \partial x \\
& -\frac{\lg (x+(n+1) d)}{2}+\frac{\lg x}{2} \\
& +\frac{d}{12} \cdot \frac{\partial \lg (x+(n+1) d)}{\partial x}-\frac{d}{12} \frac{\partial \lg x}{\partial x} \\
& -\frac{d^{3}}{120} \cdot \frac{\partial^{3} \lg (x+(n+1) d)}{1.2 .3(\partial x)^{3}}+\frac{d^{3}}{120} \cdot \frac{\partial^{3} \lg x}{1.2 .3(\partial x)^{3}}
\end{aligned}
$$

Nach Ausführung der angezeigten Operationen ergiebt sich, wenn $n-1$ statt $n$ gesetzt wird, folgender Ausdruck:

3) $\lg x^{n \mid d}=\frac{x+n d}{d} \lg (x+n d)-\frac{x+n d}{d}-\frac{x \lg x}{d}+\frac{x}{d}$

$$
\begin{array}{lc}
-\frac{\lg (x+n d)}{2} & +\frac{\lg x}{2} \\
+\frac{d}{12(x+n d)} & -\frac{d}{12 \cdot x} \\
-\frac{d^{3}}{360 \cdot(x+n d)^{3}} & +\frac{d^{3}}{360 \cdot x^{3}} \\
+\frac{d^{5}}{1260(x+n d)^{5}} & -\frac{d^{5}}{1260 x^{b}}
\end{array}
$$

In dieser Gleichung hat der letzte Factor der Facultät die Form $x+(n-1) d$. Man kann nun, um Uebereinstimmung auf beiden Seiten hervorzubringen, $\lg (x+n d)$ hinzuzählen. Dann geht (3.) über in: 
4) $\quad \lg x^{n+11 d}=\frac{x+n d}{d} \lg (x+n d)-\frac{x+n d}{d}-\frac{x \lg x}{d}+\frac{x}{d}$

$$
\begin{array}{ll}
+{ }_{2}^{1} \lg (x+n d) & +\frac{1}{2} \lg x \\
+\frac{d}{12(x+n d)} & -\frac{d}{12 x} \\
-\frac{d^{3}}{360(x+n d)^{3}} & +\frac{d^{3}}{360 x^{3}} \\
+\frac{d^{5}}{1260(x+n d)^{6}} & -\frac{d^{5}}{1260 x^{5}}
\end{array}
$$

Die Darstellung des Logarithmen einer Facultät beruht nach (3. und 4.) auf zwei Reihen, deren Glieder in (3.) unter sich correspondiren. Die Glieder der zweiten Reihe werden aus denen der ersten abgeleitet, wenn man $n=0$ setzt und ihnen entgegengesetzte Zeichen giebt. Die Glieder der Reihen in den Gleichungen (3. und 4.) stehen, mit Ausnahme des dritten, in dem nämlichen Zusammenhange. Diese Reihen sollen nun der Kürze wegen durch folgende Zeichen angedeutet werden:

$$
\begin{aligned}
& \text { 5) } \lg x^{n^{\prime} d}=R\left(\frac{x+n d}{d}\right)-R\left(\frac{x}{d}\right) \\
& \text { 6) } \lg x_{1}^{n+1}{ }^{\prime} d=F\left(\frac{x+n d}{d}\right)-R\left(\frac{x}{d}\right) .
\end{aligned}
$$

Der Zusammenhang zwischen beiden Gleichungen ist

$$
\begin{aligned}
\text { 7) } \lg x^{n+1 \mid d} & =\lg (x+n d)+R\left(\frac{x+n d}{d}\right)-R\left(\frac{x}{d}\right) \\
\text { 8) } \lg x^{n \mid d} & =F\left(\frac{x+n d}{d}\right)-\lg (x+n d)-R\left(\frac{x}{d}\right) .
\end{aligned}
$$

Die Werthberechnung des Logarithmen einer Facultät hängt demnach von der Convergenz der in den vorstehenden Gleichungen enthaltenen Reihen ab. Die Reihen convergiren sehr stark, wenn $x$ oder $x+n d$ der Zunahme gegenüber eine nicht ganz unbedeutende Zahl ist, und dann wird der gesuchte Werth leicht gefunden. Bei Facultäten von der Form $x^{n \mid d}$, wo $x, n$ und $\boldsymbol{d}$ ganze Zahlen sind, wird die erste Reihe immer stark convergiren. Ist aber die Zunahme 1 und der Grundfactor 1, wie es gewöhnlich der Fall ist, so convergiren die Glieder der zweiten Reihe nicht sehr stark, und es ist gerade für diesen Fall wichtig, den Werth der zweiten Reihe zu finden; so wie überhaupt schon aus dem Grunde, dass nach (13. §. 11.) jede Facultät von der vorliegenden Form auf eine andere gebracht werden kann, deren Basis und 
Zunahme die Einheit ist. Um nun diesen Werth zu ermitteln, setzen wir in (4.) $n-1$ statt $n, d=1$ und $x=1$. Dies giebt

9) $\lg 1^{n \mid 1}=n \lg n-n+\frac{1}{2} \lg n+\frac{1}{12 n}-\frac{1}{360 n^{3}}+\frac{1}{1260 n^{3}}+\ldots$ $-R(1)$.

Ist hier $n$ unendlich gross, so verschwinden die Glieder in $R(n)$ vom vierten an und es wird, wenn man diesen Werth von $n$ wie früher durch $a$ bezeichnet:

10) $\lg \mathbf{1}^{\alpha \mid 1}=\alpha \cdot \lg \alpha-\alpha+\frac{1}{2} \lg \alpha-R(1)$,

11) $F(\alpha)=\left(\alpha+\frac{1}{2}\right) \lg \alpha-\alpha$,

oder anders:

$$
\text { 12) } \quad \mathbf{1}^{\alpha \mid 1}=\frac{\alpha^{\alpha} \cdot V^{-}}{e^{\alpha} \cdot e^{R(1)}} \text {. }
$$

Nehmen wir nun die Gleichung (34. §. 13.) zu Hülfe und setzen dort $r$ gleichfalls unendlich gross, so erhalten wir, mit Rücksicht auf (29. §. 12.):

$$
\frac{\mathbf{1}^{\alpha \mid 1} \cdot \mathbf{1}^{\kappa \mid 1}}{\sqrt{\bar{a}}} \cdot 2^{2 \kappa}=\sqrt{\pi} \cdot \mathbf{1}^{2 \alpha \mid 1} \text {. }
$$

Werden die Facultäten dieser Gleichung nach (12.) behandelt, und wird in (12.), wegen $1^{2 \alpha \mid 1}, 2 \alpha$ statt $\alpha$ gesetzt, so findet sich

$$
\frac{\alpha^{\alpha} \sqrt{\alpha}}{e^{\alpha} e^{R_{(1)}}} \times \frac{\alpha^{\alpha} \sqrt{\alpha}}{e^{\alpha} \cdot e^{R_{(1)}}} \cdot \frac{2^{2 \alpha}}{\sqrt{\bar{\alpha}}}=\sqrt{\pi} \cdot \frac{(2 \alpha)^{2 \alpha} \sqrt{\overline{2 \alpha}}}{e^{2 \alpha} \cdot e^{h_{(1)}}} .
$$

Hieraus ist

$$
\text { 13) } \frac{1}{e^{R(1)}}=\sqrt{2 \pi} \text {, }
$$

oder

Demnach ist aus (9.)

$$
\text { 14) }-R(1)=\frac{1}{2} \lg (2 \pi) \text {. }
$$

15) $\lg 1^{n \mid 1}=\left(n+\frac{1}{2}\right) \lg n-n+\frac{1}{2} \lg 2 \pi+\frac{1}{12 n}-\frac{1}{360 n^{3}}+\frac{1}{1260 n^{5}}-\ldots$

Ist nun der Werth von $R(1)$ gefunden, so lassen sich durch ihn die Werthe von $\boldsymbol{R}_{(2)}, \boldsymbol{R}_{(3)} \ldots$ leicht berechnen. Es ist nämlich

also auch

$$
(x+n d)^{p \mid d}=\frac{x^{n \mid d}(x+n d)^{p_{i} d}}{x^{n i d}}=\frac{x^{n+p ! d}}{x^{n i d}}
$$

$$
\lg (x+n d) p \mid d=\lg x^{n+p \mid d}-\lg x^{n \mid d} .
$$

Werden die beiden ersten Ausdrücke dieser Gleichung nach (5.) behandelt, so ergiebt sich

$$
\text { 16) } \quad R\left(\frac{x+n d}{d}\right)=R\left(\frac{x}{d}\right)+\lg x^{n n^{\prime} d} \text {. }
$$


Wird hierin $x=1, z-1$ statt $n$ und $d=1$ gesetzt, so ist nach (14.):

17) $\quad R(z)=R(1)+\lg 1^{z-1 \mid 1}=-\frac{1}{2} \lg (2 \pi)+\lg 1^{z-1 \mid 1}$.

Aus dieser Gleichung können leicht die oben bemerkten Werthe berechnet werden.

Wird der Logarithme einer Facultät von der Form $x^{n_{1}^{1}-d}$ verlangt, so nehmen die Gleichungen (3. - 6.) folgende Gestalt an:

$$
\begin{aligned}
& \text { 18) } \lg x^{n \mid-d}=R\left(\frac{x-n d}{-d}\right)-R\left(\frac{x}{-d}\right) \text {, } \\
& \text { 19) } \lg x^{n+1 \mid-d}=F\left(\frac{x-n d}{-d}\right)-R\left(\frac{x}{-d}\right) \text {. }
\end{aligned}
$$

Danach wird sich die Werthbestimmung ausführen lassen, und zwar ohne Schwierigkeiten, so lange $(x-(n-1) d)$ positiv bleibt. Man kann in diesem Falle die Facultät auch umkehren und so darstellen:

$$
x^{n \mid-d}=(x-(n-1) d)(x-(n-2) d) \ldots(x-d) x .
$$

Dann tritt in (3.) $x-(n-1) d$ an die Stelle von $x$ und $x$ an die Stelle von $x+n d$.

Werden aber einzelne Glieder in $x^{n-d}$ negativ., so kann man zur Vermeidung der Logarithmen negativer Grössen die Facultät so umformen, dass sich erkennen lässt, ob sie einen positiven oder negativen Werth bekomme, und darauf die Facultät in dieser veränderten Gestalt berechnen. Hiebei kann es kommen, dass eine der Gleichungen (3. und 4.) wiederholt angewendet werden muss.

Die bisher gefundenen Gleichungen sind allgemein und gelten für jeden Werth von $n$. Man erhält sofort aus (16.) für ein negatives ganzes oder gebrochenes $n$ :

$$
R\left(\frac{x-n d}{d}\right)=R\left(\frac{x}{d}\right)+\lg \frac{1}{(x-n d)^{n \mid d}}=R\left(\frac{x}{d}\right)=\lg \frac{1}{(x-d)^{n \mid-d}}
$$

21) $\quad R\left(\frac{m x^{+n d}}{m d}\right)=R\left(\frac{x}{d}\right)+\lg x^{\frac{n}{m} \mid d}$

23) $\quad R\left(\frac{m x-n d}{m d}\right)=R\left(\frac{x}{d}\right)+\lg x^{\frac{n}{m} \mid-d}=R\left(\frac{x}{d}\right)+\lg \frac{1}{\left(x-\frac{n}{d} d\right)^{\frac{n}{m} \mid d}}$.

Wird hierin $d=1$ gesetzt, so geht (20.) über in:

$$
\text { 23) } R(x-n)=R(x)+\lg \frac{1}{(x-1)^{n ! 1}} \text {. }
$$

Setzt man nun ferner $x=1$, so zeigt sich, dass alle Werthe von $\boldsymbol{R}$ für Facultäten mit negativem Exponenten, der eine ganze Zahl ist, und deren 
Basis und Zunahme die Einheit ist, unendlich gross werden. Setzt man in (21. und 22.) $d=1, \frac{n}{m}=p+\frac{n}{m}$, so geben die beiden Gleichungen:

24) $\quad R\left(x+p+\frac{n}{m}\right)=R(x)+\lg x^{p+\frac{n}{m} \mid 1}=R(x)+\lg x^{\frac{n}{m} \mid 1}+\lg \frac{(x m+n)^{p \mid m}}{m^{p}}$

25) $\boldsymbol{R}\left(x-p-\frac{n}{m}\right)=\boldsymbol{R}(x)+\lg x^{-\frac{n}{m}-p^{11}}=\boldsymbol{R}(x)+\lg x^{-\frac{n}{m} \mid}+\lg \frac{m^{p}}{(x m-n)^{\rho^{\mid n}}}$.

Führt man in (17.) statt $z$ allmälig die Werthe 2, 3, $4 \ldots$ ein, so ergiebt sich 26) $\quad R(2)=-\frac{1}{2} \lg 2 \pi$, $R(3)=-\frac{1}{2} \lg 2 \pi+\lg (2)$, $R(4)=-\frac{1}{2} \lg 2 \pi+\lg (1 \cdot 2.3)$, $R(5)=-\frac{1}{2} \lg 2 \pi+\lg (1 \cdot 2 \cdot 3 \cdot 4)$,

Setzt man in (24.) $x=1, \frac{n}{m}=\frac{1}{2}$ und statt $p$ allmälig die Werthe $0,1,2, \ldots$, und erwägt, dass nach (25. §. 13.) $R(1)+\lg 1^{\frac{1}{2} 1}=-\frac{1}{2} \lg (2 \pi)$ $\lg \frac{1}{2}+\frac{1}{2} \lg \pi=-\frac{3}{2} \lg 2$ ist, so ergiebt sich:

27)

$$
\begin{aligned}
& R\left(\frac{3}{2}\right)=-\frac{3}{2} \lg 2 \\
& R\left(\frac{5}{2}\right)=-\frac{3}{2} \lg 2+\lg \frac{3}{2} \\
& R\left(\frac{7}{2}\right)=-\frac{3}{2} \lg 2+\lg \left(\frac{3 \cdot 5}{2 \cdot 2}\right) \\
& R\left(\frac{9}{2}\right)=-\frac{3}{2} \lg 2+\lg \left(\frac{3 \cdot 5.7}{2.2 .2}\right)
\end{aligned}
$$

Setzt man die nämlichen Werthe in (25.) und bemerkt, dass nach (26. §. 13.) $R(1)+\lg 1^{-\frac{1}{2} \mid 1}=-\frac{1}{2} \lg 2 \pi+\frac{1}{2} \lg \pi=-\frac{1}{2} \lg 2$ ist, so ergiebt sich:

$$
\text { 28) } \begin{aligned}
& R\left(\frac{1}{2}\right)=-\frac{1}{2} \lg 2 \\
& R\left(-\frac{1}{2}\right)=-\frac{1}{2} \lg 2+\lg 2 \\
& R\left(-\frac{3}{2}\right)=-\frac{1}{2} \lg 2+\lg \left(\frac{2.2}{1.3}\right) \\
& R\left(-\frac{5}{2}\right)=-\frac{1}{2} \lg 2+\lg \left(\frac{2.2 .2}{1.3 .5}\right) \\
& R\left(-\frac{7}{2}\right)=-\frac{1}{2} \lg 2+\lg \left(\frac{2.2 .2 .2}{1.3 .5 .7}\right)
\end{aligned}
$$

Soll hiernach der Werth der Facultät $1^{4+\frac{1}{2} 1}$ gefunden werden, so erhält man sofort 


$$
\begin{aligned}
\lg 1^{\frac{9}{21}} & =R\left(\frac{11}{2}\right)-R(1)=-\frac{3}{2} \lg 2+\lg 945-\lg 16+\frac{1}{2} \lg 2 \pi \\
& =1,7188567=\lg 52,34277 .
\end{aligned}
$$

Diese Zahl findet sich auch durch directe Berechnung und es ist

$$
\begin{aligned}
& 1^{\frac{9}{2 \mid} 1}=1.2 .3 .4 .5^{\frac{1}{2} \mid 1}=1.2 .3 .4 / 5\left(1-\frac{1}{2^{3} .5}+\frac{1}{2^{7} .5^{3}}+\frac{5}{2^{10} .5^{5}}-\frac{21}{2^{15} \cdot 5^{4}}-\ldots\right) \\
& =24 . / 5(1-0,025+0,00031125+0,000039625-0,000001025390625 \ldots . \\
& -0,0000004870605+0,0000000132598 \ldots) \\
& =24.2,236067977 \ldots \times 0,9753506 \ldots=52,34277 \ldots .
\end{aligned}
$$

Die Werthe, welche zu den angezeigten Operationen nöthig werden, sind fur die hyperbolische Logarithmen:

$$
\frac{1}{2} \lg 2 \pi=0,91893853320467274178
$$

und für die gemeinen Logarithmen:

$$
\frac{1}{2} \lg 2 \pi=0,399089934179057 \ldots .
$$

Bei der Reduction der hyperbolischen Logarithmen auf gemeine ist bekanntlich die Zahl 0,434294481903251827651 nöthig.

Eine ganz einfache Methode, den Werth der Function $R$ zu berechnen, geben die Gleichungen (5. und 6.) unmittelbar. $Z_{\mathrm{s}}$ ist nämlich

$$
\begin{aligned}
& \text { 29) } \quad R\left(\frac{x}{d}\right)=R\left(\frac{x+n d}{d}\right)-\lg x^{n \mid d}, \\
& \text { 30) } \quad R\left(\frac{x}{d}\right)=F\left(\frac{x+n d}{d}\right)-\lg x^{n+1 \mid d} .
\end{aligned}
$$

Hiernach sind $R\left(\frac{x+n d}{d}\right)$ und $F\left(\frac{x+n d}{d}\right)$ in Reihen zu entwickeln und von den erhaltenen Werthen dic Logarithmen der Facultäten $x^{n \mid d}$ und $x^{n+1 \mid d}$ abzuziehen. Dieses Verfahren führt sehr schnell zum Ziele, da $n$ so angenommen werden kann, dass die Reihe schnell convergirt; was schon für $n=9$ geschieht. Von der Zweckmässikeit des Verfahrens kann man sich im 6ten Bande dieses Journals S. 141 überzeugen, wo sie von mir benutzt wurde.

Die Gleichungen (3. und 4.) sind schon von Kramp (Anal. d. réfr. ast. Pg. 101) und von Bessel (Königsberger Archiv) behandelt worden. Kramp hat bei seinen Entwicklungen die Form (4.) gewählt, die Glieder der beiden Reihen

$$
\begin{aligned}
& -\frac{x+n d}{d}+\frac{d}{12(x+n d)}-\frac{d^{3}}{360(x+n d)^{3}}+\frac{d^{5}}{1260(x+n d)^{5}}-\ldots \text { und } \\
& +\frac{x}{d}-\frac{d}{12 x}+\frac{d^{3}}{360 x^{3}}-\frac{d^{5}}{1260 x^{5}}+\ldots
\end{aligned}
$$

besonders untersucht und sie durch $\Gamma \frac{d}{x+n d}$ und $\Gamma \frac{d}{x}$ bezeichnet. Die Un- 
tersuchung kann zwar auch auf diese Weise geführt werden, jedoch, wie es scheint, weniger zweckmässig; wie es auch schon Bessel bemerkt hat. Den Zusammenhang zwischen der von Kramp angenommenen Bezeichnung und der hiesigen drücken folgende Gleichungen aus:

$$
\begin{aligned}
& R\left(\frac{x+n d}{d}\right)=\frac{x+n d}{d} \lg (x+n d)-\frac{1}{2} \lg (x+n d)+\Gamma \frac{d}{x+n d} \text { und } \\
& R\left(\frac{x}{d}\right)=-\frac{x \lg x}{d}+\frac{1}{2} \lg x+\Gamma \frac{x}{d} .
\end{aligned}
$$

\section{§. 22.}

Es ist nöthig, die Werthe der Facultäten mit gebrochenen Exponenten auf eine leichte Weise finden zu können. Eine sehr einfache Mehode, den Logarithmen einer solchen Facultät darzustellen, ergiebt sich aus der Gleichung (22. §. 7.). Sie giebt

$$
a^{\frac{n}{m} \mid d}=\frac{a^{\frac{n}{m}+r \mid d}}{\left(a+\frac{n}{m} d\right)^{r \mid d}}=\frac{m^{r} \cdot a^{\frac{n+m r}{m}} \mid d}{(a m+n d)^{r \mid m d}}
$$

und hieraus

$$
\text { 1) } \lg a^{\frac{n}{m} \mid d}=\lg m^{r}-\lg (a m+n d)^{r \mid m d}+\lg a^{\frac{n+m r}{m} \mid d} \text {. }
$$

Die Werthe der zwei ersten Ausdrücke rechts vom Gleichheitszeichen lassen sich in der obigen Form durch Logarithmen darstellen und der dritte nach der Gleichung (3. §. 21.) in eine Reihe entwickeln. Hiernach ist

2) $\lg a^{\frac{n}{m} \mid d}=r \lg m-\lg (a m+n d)^{r \mid m d}+R\left(\frac{a m+n d+m r d}{m d}\right)-R\left(\frac{a}{d}\right)$.

Der entwickelte Ausdruck selbst ist

3) $\lg a^{\frac{n}{m} \mid d}=r \lg m-\lg (a m+n d)^{r \mid m d}-R\left(\frac{a}{d}\right)$

$$
\begin{aligned}
& +\frac{a m+n d+m r d}{m d} \lg \left(\frac{a m+n d+m r d}{m}\right)-\frac{a m+n d+m r d}{m d}-\frac{1}{2} \lg \left(\frac{a m+n d+m r d}{m}\right) \\
& +\frac{m d}{12(a m+n d+m r d)} \\
& -\frac{m^{3} d^{3}}{360(a m+n d+m r d)^{3}} \\
& +\frac{m^{5} d^{5}}{1260(a m+n d+m r d)^{5}} \\
& -\frac{m^{7} d^{7}}{1680(a m+n d+m r d)^{7}}
\end{aligned}
$$


Da hier die Grösse $r$ willkührlich angenommen werden kann, so ist es, wie man sieht, leicht, der begleitenden Reihe jede beliebige Convergenz zu geben. Die Gleichungen (1. bis 3.) gelten für positive wie für negative Exponenten und geben

4) $\lg a^{-\frac{n}{m} \mid d}=r \lg m-\lg (a m-n d)^{r \mid m d}+R\left(\frac{a m-n d+r m d}{m d}\right)-R\left(\frac{a}{d}\right)$, und hieraus nach (3. \$. 21.)

5) $\quad \lg a^{-\frac{n}{m} \mid d}=r \lg m-\lg (a m-n d)^{r \mid m d}-R\left(\frac{a}{d}\right)$

$$
\begin{aligned}
& +\frac{a m+m r d-n d}{m d} \lg \left(\frac{a m-n d+r m d}{m}\right)-\frac{1}{2} \lg \left(\frac{a m+r m d-n d}{m}\right)=\frac{a m+r m d-n d}{m d} \\
& +\frac{m d \cdot}{12(a m+m r d-n d)} \\
& -\frac{m^{3} d^{3}}{360(a m+m r d-n d)^{5}} \\
& +\frac{m^{5} d^{5}}{1260(a m+m r d-n d)^{7}}
\end{aligned}
$$

Die vorstehenden Gleichungen werden einfacher, wenn man Basis und Zunahme $=1$ setzt. Es ist alsdann

6) $\left.\mathbf{1}^{\frac{n}{m} \mid}\right|^{1}=r \lg m-\lg (m+n)^{r_{1} m}+\frac{1}{2} \lg 2 \pi$

$$
\begin{aligned}
& +\frac{m(r+1)}{m}+n \\
& +\frac{m}{\ln \left(\frac{m(r+1)+n}{m}\right)-\frac{1}{2} \lg \left(\frac{m(r+1)+n}{m}\right)-\frac{m(r+1)+n}{m}} \\
& +\frac{m^{3}}{360(m(r+1)+n)^{3}}+\frac{m^{5}}{1260(m(r+1)+n)^{5}}-\frac{m^{7}}{1680(m(r+1)+n)^{7}}+\ldots
\end{aligned}
$$

7) $\quad 1^{-\frac{n}{m} \mid 1}=r \lg m-\lg (m-n)^{r \mid m}+\frac{1}{2} \lg 2 \pi$

$$
\begin{aligned}
& +\frac{m(r+1)-n}{m} \lg \left(\frac{m(r+1)-n}{m}\right)-\frac{1}{2} \lg \left(\frac{m(r+1)-n}{m}\right)-\frac{m(r+1)-n}{m}-n \\
& +\frac{m}{12(m(r+1)-n)}-\frac{m^{3}}{360(m(r+1)-n)^{3}}+\frac{m^{5}}{1260(m(r+1)-n)^{5}}-\frac{m^{7}}{1680(m(r+1)-n)^{7}}+\ldots
\end{aligned}
$$

Die Brauchbarkeit dieser Gleichungen soll an besondern Fällen gezeigt werden. Wir wählen hiezu die Darstellung des schon früher gefundenen Werths von $1^{\frac{1}{2} \mid 1}$. Setzt man in (6.) $r=9, n=1, m=2$, so ergiebt sich

8) $\lg 1^{\frac{1}{2} \mid 1}=9 \lg 2-\lg 3^{912}+\frac{1}{2} \lg 2 \pi+\frac{21}{2} \lg \frac{21}{2}-\frac{1}{2} \lg \frac{21}{2}$

$$
-\frac{21}{2}+\frac{2}{12.21}-\frac{2^{3}}{360.21^{3}}+\frac{2^{5}}{1260.21^{5}}-\frac{2^{7}}{1680.21^{7}}+\ldots
$$

Werden hier die angezeigten Rechnungen ausgeführt und die Werthe der Logarithmen gesetzt, so erhält man 
7. Oettinger, Untersuchungen üler die analytischen Facultütèn.

$$
\begin{gathered}
\lg 1^{\frac{\mathrm{T}}{2} 1}=2,7092700-8,8160616+10,7224876+0,3990899 \\
-10,5+0,00793650793 \ldots+0,0000023995488 \ldots \\
-0,0000000062184 \ldots
\end{gathered}
$$

Die Glieder der Reihe geben den hyperbolischen Logarithmen. Ihr Werth ist

$$
K=-10,492065855 \ldots
$$

Der Briggische Logarithme ist

$$
K_{1}=-4,556646315 \ldots
$$

Werden nun die Werthe der vorstehenden Logarithmen zusammengenommen, so erhält man

$$
1^{\frac{1}{2} 1}=N 0,9475449-1=0,8862269 \ldots
$$

Dieser Werth wurde schon in $(\$$. 15.) gefunden.

Die Methode lässt sich ebensowohl benutzen, wenn der Exponent einer Facultät einen kleinen Bruch bedeutet. Ist z. B. der Logarithme von $1^{\frac{1}{100} \mid 1} \mathrm{zu}$ berechnen, so setze man in (6.) $r=8, m=100, n=1$. Dies giebt

9) $\lg 1^{\frac{1}{100} \mid 1}=8 . \lg 100-\lg 101^{81100}+\frac{1}{2} \lg 2 \pi+9,01 \lg 9,01-\frac{1}{2} \lg 9,01$

$$
-9,01+\frac{100}{12.901}-\frac{100^{3}}{360.901^{3}}+\frac{100^{6}}{1260.901^{5}}-\frac{100^{7}}{1680.901^{7}}+\ldots
$$

Werden die angezeigten Glieder berechnet, so findet sich

$$
\begin{gathered}
\lg 1^{\frac{1}{10} \mid 1}=16-20,6172911+0,3990899+8,6020703 \ldots .0,4773623 \\
-9,01+0,0092489826 \ldots-0,000003797721 \ldots+0,000000013365 \ldots . \\
-0,00000000012348 \ldots .
\end{gathered}
$$

Der Werth des hyperbolischen Logarithmen der Reihe ist

$$
K=-9,000754801867 \ldots
$$

Der Briggische Logarithme ist

$$
K_{1}=-3,9089781 \text {. }
$$

Durch Ausführung der angezeigten Rechnung erhält man

$$
1^{\frac{1}{100} \mid 1}=N 0,9975287-1=0,9943257 \ldots .
$$

Die Pechnung ist mit siebenstelligen Logarithmen gemacht. Die Ermitlung des Werths des hyperbolischen Logarithmen der begleitenden Reihe macht viele Mühe, wenn grosse Zahlen, wie in (9.) vorkommen. Man kann sich diese Rechnungen erleichtern, wenn man nur in dem ersten und höchstens in dem zweiten Gliede die Division wirklich ausführt, die Werthe der spätern Glieder aber durch Logarithmen sucht. Dann werden selbst siebenstellige Logarithmen genügen, weil die Glieder der Reihe stark convergiren. Die Gleichungen (6. und 7.) würden vollkommen genügen, wenn $(m(r+1)+n)$ immer so angenom- 
men werden könnte, dass sich die Zahl 10 oder ein Vielfaches von 10 ergäbe. Dies geht aber, wie leicht $\mathrm{zu}$ sehen, nicht an. Es ergiebt sich jedoch aus den Gleichungen (3. und 5.) noch ein anderes Mittel, stark convergirende Reihen abzuleiten. Die Glieder der Reihe in diesen Ausdrücken lassen sich nämlich nach dem binomischen Lehrsatze in Reihen entwickeln. Bezeichnen wir der Reihe nach die Vorzahlen $\frac{1}{12}, \frac{1}{360}, \frac{1}{1260}, \ldots$ der Kürze wegen durch $a_{1}, a_{2}, a_{3}, a_{4} \ldots$, so erhalten wir

$$
\begin{gathered}
a_{1} \frac{m d}{((a+r d) m+n d)}=\frac{a_{1} d}{a+r d}-\frac{a_{1} n d^{2}}{(a+r d)^{2} m}+\frac{a_{1} d^{3}}{(a+r d)^{3}} \cdot \frac{n^{2}}{m^{2}}-\frac{a_{1} d^{4}}{(a+r d)^{4}} \cdot \frac{n^{3}}{m^{3}}-\frac{a_{1} d^{5}}{(a+r d)^{5}} \cdot \frac{n^{4}}{m^{4}}+\ldots \\
-a_{3} \frac{m^{3} d^{3}}{((a+r d) m+n d)^{3}}=-\frac{a_{3} d^{3}}{(a+r d)^{3}}+[2]_{2} \cdot \frac{a_{3} d^{4}}{(a+r d)^{4}} \frac{n}{m}-[3]_{2} \cdot \frac{a_{3} d^{5}}{(a+r d)^{5}} \frac{n^{2}}{m^{2}}+[4]_{2} \cdot \frac{a_{3} d^{6}}{(a+r d)^{6}}\left(\frac{n}{m}\right)^{3}+\ldots \\
+\frac{a_{5} m^{5} d^{5}}{((a+r d) m+n d)^{5}}=\frac{a_{5} d^{5}}{(a+r d)^{5}}-[2]_{4} \frac{a_{5} d^{6}}{(a+r d)^{6}} \cdot \frac{n}{m}+[3]_{4} \frac{a_{5} d^{7}}{(a+r d)^{7}} \frac{n^{2}}{m^{2}}-[4]_{4} \cdot \frac{a_{5} d^{5}}{(a+r d)^{8}} \cdot \frac{n^{3}}{m^{3}}+\ldots \\
-\frac{a_{7} m^{7} d^{7}}{((a+r d) m+n d)^{7}}=-\frac{a_{7} d^{7}}{(a+r d)^{7}}+[2]_{6} \frac{a_{7} d^{8}}{(a+r d)^{8}} \frac{n}{m}-[3]_{6} \frac{a_{7} d^{9}}{(a+r d)^{9}} \frac{n^{2}}{m^{2}}+[4]_{6} \frac{a_{7} d^{10}}{(a+r d)^{10}} \cdot\left(\frac{n}{m}\right)^{3}+\ldots \\
+\frac{a_{9} m^{9} d^{9}}{((a+r d) m+n d)^{9}}=\frac{a^{9} d^{9}}{(a+r d)^{9}}-[2]_{8} \frac{a^{9} d^{10}}{(a+r d)^{10}} \frac{n}{m}+[3]_{8} \frac{a_{9} d^{11}}{(a+r d)^{11}} \cdot \frac{n^{2}}{m^{2}}-[4]_{8} \frac{a^{11} d^{12}}{(a+r d)^{12}} \cdot \frac{n^{3}}{m^{3}}+\ldots
\end{gathered}
$$

Ordnet man diese Reihen nach den Potenzen von $\frac{n}{m}$, so erhält man sehr stark convergirende Reihen und der Ausdruck (3.) geht in folgenden über:

10) $\lg a^{\frac{n}{m} \mid d}=r \lg m-\lg (a m+n d)^{r \mid m d}-R\left(\frac{a}{d}\right)$

$$
\begin{aligned}
& +\frac{m(a+r d)+n d}{m d} \lg \left(\frac{m(a+r d)+n d}{m}\right)-\frac{m(a+r d)+n d}{m d}-\frac{1}{2} \lg \left(\frac{m(a+r d)+n d}{m}\right) \\
& +\left(\frac{n}{m}\right)^{0}\left(\frac{a_{1} d}{a+r d}-\frac{a_{3} d^{3}}{(a+r d)^{3}}+\frac{a_{5} d^{5}}{(a+r d)^{5}}-\frac{a_{7} d^{7}}{(a+r d)^{7}}+\ldots\right) \\
& -\frac{n}{m}\left(\frac{a_{1} d^{2}}{(a+r d)^{2}}-[2]_{2} \frac{a_{3} d^{4}}{(a+r d)^{4}}+[2]_{4} \frac{a_{5} d^{6}}{(a+r d)^{6}}-[2]_{6} \frac{a_{7} d^{8}}{(a+r d)^{8}}+\ldots\right) \\
& +\frac{n^{2}}{m^{2}}\left(\frac{a_{1} d^{3}}{(a+r d)^{3}}-[3]_{2} \frac{a_{3} d^{5}}{(a+r d)^{5}}+[3]_{4} \frac{a_{5} d^{7}}{(a+r d)^{7}}-[3]_{6} \frac{a_{7} d^{9}}{(a+r d)^{9}}+\ldots\right) \\
& -\frac{n^{3}}{m^{3}}\left(\frac{a_{1} d^{4}}{(a+r d)^{4}}-[4]_{2} \frac{a_{3} d^{6}}{(a+r d)^{6}}+[4]_{4} \frac{a_{5} d^{8}}{(a+r d)^{8}}-[4]_{6} \frac{a_{7} d^{10}}{(a+r d)^{10}}+\ldots\right) \\
& +\frac{n^{4}}{m^{4}}\left(\frac{a_{1} d^{5}}{(a+r d)^{4}}-[5]_{2} \frac{a_{3} d^{7}}{(a+r d)^{7}}+[5]_{4} \frac{a_{5} d^{9}}{(a+r d)^{9}}-[5]_{6} \frac{a_{7} d^{11}}{(a+r d)^{11}}+\ldots\right)
\end{aligned}
$$

Aus (5.) erhält man unmittelbar, wenn in (10.) $-n$ statt $n$ gesetzt wird: 
11) $\lg a^{-\frac{n}{m} \mid d}=r \lg m-\lg (a m-n d)^{r m d}-R\left(\frac{a}{d}\right)$

$$
\begin{aligned}
& +\frac{m(a+r d)-n d}{m d} \lg \left(\frac{m(a+r d)-n d}{m}\right)-\frac{m(a+r d)-n d}{m d}-\frac{1}{2} \lg \left(\frac{m(a+r d)-n d}{m}\right) \\
& +\left(\frac{n}{m}\right)^{0}\left(\frac{a_{1} d}{a+r d}-\frac{a_{3} d^{3}}{(a+r d)^{3}}+\frac{a_{5} d^{5}}{(a+r d)^{5}}-\frac{a_{7} d}{(a+r d)^{7}}+\ldots\right) \\
& \left.+\frac{n}{m}\left(\frac{a_{1} d^{2}}{(a+r d)^{2}}-[2]_{2} \frac{a_{3} d^{3}}{(a+r d)^{3}}+[2]_{4} \frac{a_{5} d^{6}}{(a+r d)^{6}}-[2]_{6} \frac{a_{7} d^{8}}{(a+r d)^{8}}\right)+\ldots .\right) \\
& \left.+\frac{n}{m}\left(\frac{a_{1} d^{3}}{(a+r d)^{3}}-[3]_{2} \frac{a_{3} d^{5}}{(a+r d)^{5}}+[3]_{4} \frac{a_{5} d^{7}}{(a+r d)^{7}}-[3]_{6} \frac{a_{7} d^{9}}{(a+r d)^{9}}\right)+\ldots .\right)
\end{aligned}
$$

Diese Formeln sind ganz allgemein. Sie lassen sich sogleich auf eine Facultät mit negativer Zunahme anwenden, wenn $-d$ statt $+d$ gesetzt wird. Die besondere Darstellung von $\lg a^{\frac{n}{m} \mid-d}$, und $\lg a^{-\frac{n}{m} \mid-d}$ ist nicht nöthig, da in ( $\$$. 11. und 13.) der Zusammenhang zwischen den Facultäten mit gebrochenen Exponenten bei positiver und negativer Zunahme angegeben ist. Es genügen also die in (3. und 5.) gegebenen Ausdrücke, indem sich von ihnen ohne Schwierigkeit auf die übrigen der Facultäten übergehen lässt.

Bei der Ausführung der in (10. und 11.) angezeigten Rechnungen sind die Glieder der zwei ersten Reihen durch hyperbolische oder Briggische Logarithmen unmittelbar anzugeben. Dies hat keine Schwierigkeit, denn man hat höchstens die Logarithmen einer Facultät von neun Factoren darzustellen und zusammenzuzählen. Die begleitenden Reihen führen immer auf hyperbolische Logarithmen und diese lassen sich sofort auf Briggische bringen, wenn man mit letztern rechnet. Setzt man $r=9, d=1, a=1$, so erhält man stark convergirende Reihen, deren Glieder Potenzen der Zahl 10 zu Nennern haben, und die also keiner weitern Rechnung bedürfen. So findet sich aus (10.): 
12) $\lg 1^{\frac{n}{m}}=\lg m^{9}-\lg (m+n)^{9 \mid m}+\frac{1}{2} \lg 2 \pi+\frac{10 m+n}{m} \lg \frac{10 m+n}{m}-\frac{1}{2} \lg \frac{10 m+n}{m}-\frac{10 m+n}{m}$

$$
\begin{aligned}
& +1\left(\frac{a_{1}}{10}-\frac{a_{3}}{10^{3}}+\frac{a_{5}}{10^{5}}-\frac{a_{7}}{10^{7}}+\frac{a_{9}}{10^{9}}-\frac{a_{11}}{10^{11}}+\frac{a_{13}}{10^{13}}-\frac{a_{15}}{10^{15}}+\ldots\right) \\
& -\frac{n}{m}\left(\frac{a_{1}}{10^{2}}-\frac{3 a_{3}}{10^{4}}+\frac{5 a_{5}}{10^{6}}-\frac{7 a_{7}}{10^{8}}+\frac{9 a_{9}}{10^{10}}-\frac{11 a_{11}}{10^{12}}+\frac{13 a_{13}}{10^{14}}-\frac{15 a_{15}}{10^{16}}+\ldots\right) \\
& +\frac{n^{2}}{m^{2}}\left(\frac{a_{1}}{10^{3}}-\frac{6 a_{3}}{10^{5}}+\frac{15 a_{5}}{10^{7}}-\frac{28 a_{7}}{10^{9}}+\frac{45 . a_{9}}{10^{11}}-\frac{66 . a_{11}}{10^{13}}+\frac{91 a_{13}}{10^{15}}-\frac{120 a_{15}}{10^{17}}+\ldots\right) \\
& -\frac{n^{3}}{m^{3}}\left(\frac{a_{1}}{10^{4}}-\frac{10 a_{3}}{10^{6}}+\frac{35 a_{5}}{10^{8}}-\frac{81 a_{7}}{10^{10}}+\frac{165 a_{9}}{10^{12}}-\frac{286 a_{11}}{10^{14}}+\frac{455 a_{13}}{10^{16}}-\frac{680 a_{15}}{10^{18}}+\ldots\right) \\
& +\frac{n^{4}}{m^{4}}\left(\frac{a_{1}}{10^{5}}-\frac{15 . a_{3}}{10^{7}}+\frac{70 . a_{5}}{10^{9}}-\frac{210 . a_{7}}{10^{11}}+\frac{495 . a_{9}}{10^{13}}-\frac{1001 a_{11}}{10^{15}}+\frac{1820 a_{13}}{10^{17}}-\frac{2890 a_{15}}{10^{19}}+\ldots .\right) \\
& -\frac{n^{5}}{m^{5}}\left(\frac{a_{1}}{10^{6}}-\frac{21 . a_{3}}{10^{8}}+\frac{126 a_{5}}{10^{10}}-\frac{462 a_{7}}{10^{12}}+\frac{1287 a_{9}}{10^{14}}-\frac{3003 a_{11}}{10^{16}}+\frac{6188 a_{13}}{10^{18}}-\frac{11628 a_{15}}{10^{20}}+\ldots\right)
\end{aligned}
$$

Die Ermittlung der Werthe der begleitenden Reihen erfordert allerdings viel Rechnung. Die Resultate sind aber auch sehr brauchbar. Es ist nämlich nöthig, die Zahlenwerthe von $a_{1}, a_{2}, a_{3} \ldots$ in Decimalbrüchen darzustellen, sie mit ihren Vorzahlen zu multipliciren und dann mit den gehörigen Zeichen zusammenzuzählen. Die Berechnung der Zahlenwerthe von $a_{1}$, $a_{2}, a_{3}, a_{4}, \ldots$ beruht auf den sogenannten Bernoullischen Zahlen, die in (\$. 19.) gefunden wurden und bekannt sind. Euler hat in seiner Differentialrechnung (2ter Thl. §. 132. 5tes Cap.) die funfzehn ersten dieser Zahlen angegeben. Rothe hat die sechszehn folgenden berechnet (S. d. Journ. 20ter Bd. S. 11.). Da ihr Ausdruck in Decimalbrüchen nicht unwichtig sein dürfte, so theilen wir denselben hier mit:

$$
\text { 13) } \begin{aligned}
\mathfrak{A} & =\frac{1}{6}=0,16666 \ldots \\
\mathfrak{B} & =\frac{1}{30}=0,03333 \\
\mathfrak{S} & =\frac{1}{42}=0,0238095238095 \ldots \\
\mathfrak{D} & =\frac{1}{30}=0,03333 \ldots \\
\mathfrak{E} & =\frac{5}{66}=0,07575 \ldots \\
\mathfrak{F} & =\frac{691}{2730}=0,253113553113 \ldots \\
\mathfrak{S} & =\frac{7}{6}=1,16666 \ldots \\
\mathfrak{S} & =\frac{3617}{510}=7,092156862745098039215686 \ldots
\end{aligned}
$$




$$
\begin{aligned}
& \mathfrak{I}=\frac{43867}{798}=54,971177944862155388471177944 \ldots \\
& \mathfrak{I}=\frac{174611}{330}=529,124242424 \ldots \\
& \mathfrak{L}=\frac{854513}{138}=6192,123185405797101449275362318840579 \ldots \\
& \mathfrak{T}=\frac{236364091}{2730}=86580,25311351648351648 \ldots \\
& \mathfrak{N}=\frac{8553103}{6}=1425517,16666 \ldots
\end{aligned}
$$

Man sieht, dass sämmtliche Brüche periodisch sind. Der Bruch $\mathfrak{S}$ hat eine Periode von 16, der Bruch $\mathfrak{L}$ eine Periode von 22 Stellen. Euler hat die Decimalbrüche (6tes Cap. d. Differential-Rechnung 2ter Thl. §. 144.) der acht ersten Vorzahlen mitgetheilt, hat aber nicht bemerkt, dass sie periodisch sind. Wenigstens ist die Periode des Bruches $\mathfrak{S}$ nicht von ihm angegeben. Die hiesigen Resultate stimmen mit denen von Euler überein.

Die Werthe der Zahlen, welche nun zur entwickelten Darstellung von (12.) nöthig sind, sind folgende:

$$
\text { 14) } \begin{aligned}
a_{1} & =\frac{1}{6} \cdot \frac{1}{1.2}=0,0833333 \ldots \\
a_{3} & =\frac{1}{30} \cdot \frac{1}{3.4}=0,0027777 \ldots \\
a_{5} & =\frac{1}{42} \cdot \frac{1}{5.6}=0,000793650793650 \ldots \\
a_{7} & =\frac{1}{30} \cdot \frac{1}{7.8}=0,00059523809522380 \ldots \\
a_{9} & =\frac{5}{66} \cdot \frac{1}{9.10}=0,000841750841750 \ldots \\
a_{11} & =\frac{691}{2730} \cdot \frac{1}{11.12}=0,00191752691752 \ldots \\
a_{13} & =\frac{7}{6} \cdot \frac{1}{13.14}=0,006410256410256 \ldots \\
a_{15} & =\frac{3617}{510} \cdot \frac{1}{15.16}=0,029550653594771241830 \ldots \\
a_{17} & =\frac{43867}{798} \cdot \frac{1}{17.18}=0,179644372368830573 \ldots \\
a_{19} & =\frac{174611}{330} \cdot \frac{1}{19.20}=1,392432216905901116427433221 \ldots \\
. & . . .
\end{aligned}
$$

Die spätern Zahlen sind nicht mehr nöthig, da sie nach (12.) auf die 18te Decimalstelle nicht mehr Einfluss haben.

Werden die vorstehenden Werthe nach (12.) eingeführt und zusammengezählt, so erhält man folgenden Ausdruck:

Crelle's Journal f. d. M. Bd. XXXIII. Heft 2. 
15) $\lg 1^{\frac{n}{m} \mid} \operatorname{1}=9 \lg m-\lg (m+n)^{9 \mid m}+\frac{10 m+n}{m} \lg \frac{10 m+n}{m}-\frac{1}{2} \lg \frac{10 m+n}{m}+\frac{1}{2} \lg 2 \pi$ $-\frac{10 m+n}{m}+\left(\frac{n}{m}\right)^{0} 0,0083305634333628712 \ldots$

$-\frac{n}{m} \quad 0,0008325039273245763 \ldots$

$+\frac{n^{2}}{m^{2}} \quad 0,0000831678408428730 \ldots$

$-\frac{n^{3}}{m^{3}} \quad 0,0000083058284670102 \ldots$

$+\frac{n^{4}}{m^{4}} \quad 0,0000008292210120777 \ldots$

$-\frac{n^{5}}{m^{5}} \quad 0,0000000827597352940 \ldots$

$+\frac{n^{6}}{m^{6}} \quad 0,0000000082571696073 \ldots$

- $\frac{n^{7}}{m^{7}} \quad 0,0000000008235855311 \ldots$

$+\frac{n^{8}}{m^{8}} \quad 0,0000000000821209322 \ldots$

$-\frac{n^{9}}{m^{9}} \quad 0,0000000000081859511 \ldots$.

$+\frac{n^{10}}{m^{10}} 0,0000000000008153628 \ldots$

$-\left(\frac{n}{m}\right)^{11} 0,0000000000000812682 \ldots$.

$+\left(\frac{n}{m}\right)^{12} 0,00000000000000809398 \ldots$

$-\left(\frac{n}{m}\right)^{13} 0,0000000000000008059 \ldots$

oder auch

16) $\left.\lg 1^{\frac{n}{m}}\right|^{1}=9 \lg m-\lg (m+n)^{9 \mid m}+\frac{10 m+n}{m} \lg \frac{10 m+n}{m}-\frac{1}{2} \lg \frac{10 m+n}{m}--\frac{1}{2} \lg 2 \pi$

$-9,9916694365666371287$

$-\frac{n}{m} \cdot 1,00083250392732457 \ldots$

$+\frac{n^{2}}{m^{2}} 0,0000831678408428730 \ldots$

$-\frac{n^{3}}{m^{3}} 0,00000830582846701 \ldots$

Diese Gleichungen geben die Logarithmen der Facultäten mit gebrochenen Exponenten auf eine sehr bequeme Weise; besonders dann, wenn $\frac{n}{m}$ ein kleiner Bruch ist. Der Logarithme lässt leicht auf zwölf bis funfzehn Decimalstellen berechnen und es können zu der Rechnung sowohl hyper- 
bolische als künstliche Logarithmen benutzt werden. Wir geben den Logarithmen der Facultät $\mathbf{1}^{\frac{1}{100} \mid \mathbf{l}}$ durch künstliche und durch natürliche Logarithmen. Aus der Gleichung (15.) erhalten wir

17) $\lg 1^{\frac{1}{10 \overline{0}}}=9 \lg 100-\lg 101^{9100}+\frac{1}{2} \lg 2 \pi+10,01 \lg 10,01-\frac{1}{2} \lg 10,01$

$$
\begin{aligned}
-10,01 & +0,008330563433 \ldots \\
& -0,000008325039 \ldots \\
& +0,0000000083167840 \ldots \\
& -0,0000000000083058 \ldots \\
& +0,00000000000000829 \ldots
\end{aligned}
$$

Wendet man zunächst künstliche und zehnstellige Logarithmen an, so ist die begleitende Reihe, welche $K$ genannt werden soll, aul künstliche Logarithmen z.u bringen. Es ist

$\lg$ brigg. $K=0,434294481903 \ldots \times-10,00167775.32974238 \ldots$

$$
=-4,3436734582 \ldots
$$

Werden nun die angezeigten Rechnungen ausgeführt, so erhält man

$$
\begin{aligned}
& 9 \lg 100=18,0000000000-\lg 101^{91100}=-23,5720158224 \\
& \frac{1}{2} \lg 2 \pi=0,3990899341-\frac{1}{2} \lg 10,01=-0,5002170387 \\
& 10,01 \lg 10,01=\frac{10,0143451157}{28,4134350498}-\lg \text { brigg. } K=-4,3436734582 \\
& \hline
\end{aligned}
$$

Demnach ist

18) lg. brigg. $1^{1^{1} \frac{1}{0} 1}=-0,0024712695=0,9975287305-1$.

Dieser Logarithme ist bis auf die neunte Decimalstelle richtig. Verlangt man ihn auf eine grössere Zahl von Decimalstellen, so kann man sich der natïrlichen Logarithmen bedienen. Die in (17.) angezeigten Rechnungen sind dann mit natürlichen Logarithmen zu machen. Man erhält, wenn man sechszechn Decimalstellen nimmt, aus (17.):

$$
\begin{aligned}
9 \lg 100 & =41,4465316738928223 \\
\frac{1}{2} \lg 2 \pi & =0,9189385332046727 \\
10,01 \lg 10,01 & =\frac{23,0588817792045634}{65,42435198631120584} \\
-\lg 101^{9100}= & -54,2765722442871397 \\
-\frac{1}{2} \lg 10,01= & -1,1517922966635646 \\
-K \quad & -10,0016777532974238 \\
& -65,4300422942481281 .
\end{aligned}
$$


Demnach ist

19) lg. nat. $1^{\frac{1}{100} \mid 1}=-0,00569030794606967 \ldots$

Bringt man diesen Werth auf künstliche Logarithmen, so findet sich

20) lg. brigg. $1^{\frac{1}{100} 1}=-0,00247126934130826 \ldots$

$$
=0,99752873065869173 \ldots-1 \text {. }
$$

In diesem Ausdruck ist noch die sechszehnte Decimalstelle richtig; wie sich aus dem folgenden Paragraphen, No. 16., ergeben wird.

Setzt man in (15.) $-n$ statt $+n$, so erhält man, mit Rücksicht auf (11.), den Logarithmen einer Facultät mit negativem Exponenten, und es ergiebt sich

21) $\lg 1^{-\frac{n}{m} \mid 1}=9 \lg m-\lg (m-n)^{g \mid m}+\frac{1}{2} \lg 2 \pi+\frac{10 m-n}{m} \lg \frac{10 m-n}{m}-\frac{1}{2} \lg \frac{10 m-n}{m}$

$$
\begin{aligned}
-\frac{10 m-n}{m} & +\left(\frac{n}{m}\right)^{0} 0,00833056343336287 \ldots \\
& +\frac{n}{m} \quad 0,00083250392732457 \ldots \\
& +\frac{n^{2}}{m^{2}} 0,0000831678408428730 \ldots \\
& +\frac{n^{3}}{m^{3}} \quad 0,00000830582846701 \ldots
\end{aligned}
$$

oder auch

22) $\lg 1^{\frac{n}{m}} \operatorname{l}=9 \lg m-\lg (m-n)^{9 \mid m}+\frac{1}{2} \lg 2 \pi+\frac{10 m-n}{m} \lg \frac{10 m-n}{m}-\frac{1}{2} \lg \frac{10 m-n}{m}$

$$
\text { - 9,99166943656663712 ... }
$$$$
+\frac{n}{m} \cdot 1,00083250392732457 \ldots
$$$$
+\frac{n^{2}}{m^{2}} 0,0000831678408428730 \ldots
$$$$
+\frac{n^{3}}{m^{3}} 0,00000830582846701 \ldots
$$

Die Glieder der Reihen (15. und 21.) convergiren, wie schon bemerkt, um so stärker, je kleiner der Bruch $\frac{n}{m}$ ist, und weniger stark, wenn $\frac{n}{m}$ der Einheit nahe kommt. Jedoch geben sie in diesem Falle den Logarithmen auf wenigstens zwölf Decimalstellen richtig an; was meistens hinreicht. Man kann jedoch auch noch mehr Glieder der Reihe entwickeln und dann den Logarithmen auf eine beliebige Anzahl von Stellen genau finden. Dabei haben die 
Reihen den Vortheil, dass sie die Summirung von nicht mehr als neun Logarithmen erfordern, welches auch der Exponent sei. Selbst noch, wenn sich der Exponent $\frac{n}{m}$ bis zu $\frac{1}{2}$ erhebt, geben die Reihen den Logarithmen auf wenigstens zwölf Decimalstellen richtig an. Die Ausdrücke (15. und 21.) haben ausserdem noch die Eigenschaft, dass sie sich gegenseitig ergänzen, etwa wic Sinus und Cosinus, und dadurch die Darstellung des Logarithmen einer Facultät sehr leicht geben, wenn auch der Exponent $\frac{n}{m}$ der Einheit nahe liegt.

Für diesen Fall sei $\frac{p}{m}$ die Ergänzung von $\frac{n}{m}$ zur Einheit, so dass $\frac{n+p}{m}=1$, also $\frac{n}{m}=1-\frac{p}{m}$ ist. Dann ist

$$
\mathbf{1}^{\left.\frac{n}{m}\right|^{1}}=1^{-\frac{p}{m}+1 \mid 1}=1^{-\frac{p}{m} \mid 1}\left(1-\frac{p}{m}\right)=\frac{n}{m} \cdot 1^{-\left.\frac{p}{m}\right|_{1} ^{1}} .
$$

Wird nun $1^{-\frac{p}{n} \mid 1}$ nach (21.) berechnet (was jetzt leicht geschehen kann, da $\frac{p}{m}$ ein kleiner Bruch ist) und wird hievon $\frac{n}{m}$ genommen, so ist dadurch der Werth von $1^{\frac{n}{m}} \mid \mathbf{1}$ gefunden. Es ergicbt sich für die angedeutete Zurückführung folgende Gleichung:

23) $\lg \mathbf{1}^{\frac{n}{m} \mid \mathrm{t}}=\lg \mathbf{1}^{-\frac{p}{m} \mid}+\lg n-\lg m$.

Eben so ist unter der nämlichen Voraussetzung, wenn $\frac{n}{m}$ der Einheit nahe liegt, umgekehrt:

$$
1^{-\frac{n}{m} \mid 1}=1^{-1+\frac{p}{m} \mid 1}=1^{\frac{p}{m} \mid 1}\left(1+\frac{p}{m}\right)^{-1 \mid 1}=1^{\frac{p}{m} \mid 1} \cdot \frac{m}{p},
$$

also auch

24) $\lg \mathbf{1}^{-\frac{n}{m} \mid}=\lg \mathbf{1}^{\frac{p}{m} \mid}+\lg m-\lg p$.

Nach diesen Gleichungen lassen sich Logarithmentafeln für Facultälen mit gebrochenen Exponenten, etwa von $1^{011}$ bis $1^{1 \mid 1}$, construiren. Dabei hommen die vier Grundformen $1^{\frac{n}{m} \mid 1}, \mathbf{1}^{-\frac{n}{m} \mid}, \mathbf{1}^{\frac{n}{m} \mid-1} \cdot \mathbf{1}^{-\frac{n}{m} \mid-1}$ in Betracht. Es genügt jedoch eine Tafel für die eine Grundform, weil aus ihr, wie gezeigt, die übrigen abgeleitet werden können. Die Hülfsgleichungen, welche dazu dienen sind, ausser den in (23. und 24.) angegebenen, nach (\$. 13.) folgende:
25)$$
\lg \mathbf{1}^{\frac{n}{m_{i}}-1}=\lg m-\lg (m-n)-\lg \mathbf{1}^{-\frac{n}{m} \mid},
$$$$
\lg 1^{-\frac{n}{m} \mid-1}=\lg m-\lg (m+n)-\lg 1^{\left.\frac{n}{m}\right|^{1}} \text {. }
$$ 
Hat man Tafeln der künstlichen Logarithmen auf eine hinlängliche Anzahl von Stellen, so kann man die Gleichungen (14. und 16.) auch dadurch noch bequemer zur Benutzung einrichten, dass man die Glieder der begleitenden Reihe mit der Zahl 0,43429481 .... multiplicirt und sie so auf künstliche Logarithmen bringt. Bei der Ausführung dieser Rechnungen sind die Gleichungen (16. und 22.) zu Grunde zu legen. Man erhält dann folgende Ausdrücke:

27) $\lg$. br. $1^{\frac{n}{m} \mid}=9 \lg m-\lg (m+n)^{9 \mid m}-\frac{10 m+n}{m} \lg \frac{10 m+n}{m}-\frac{1}{2} \lg \frac{10 m+n}{m}+\frac{1}{2} \lg 2 \pi$

$-4,339326901302263773 \ldots$.

$-\frac{n}{m} 0,434656033765051676 \ldots$

$+\frac{n^{2}}{m^{2}} 0,000036119334349867 \ldots$

$-\frac{n^{3}}{m^{3}} 0,000003607175470860 \ldots$

$+\frac{n^{4}}{m^{4}} 0,0000003601261098235$

$-\frac{n^{5}}{m^{5}} 0,0000000359420963619 \ldots$.

$+\frac{n^{6}}{m^{6}} 0,0000000035860431966 \ldots$

$-\frac{n^{7}}{m^{7}} 0,0000000003576786515 \ldots$

$+\frac{n^{8}}{m^{8}} 0,0000000000356646677 \ldots$

$-\frac{n^{9}}{m^{9}} 0,0000000000035551133 \ldots$.

$+\frac{n^{10}}{m^{10}} 0,0000000000003541032 \ldots$

$-\frac{n^{11}}{m^{11}} 0,00000000000003529843 \ldots$

$+\frac{n^{12}}{m^{12}} 0,00000000000000351517 \ldots$

$--\frac{n^{13}}{m^{13}} 0,0000000000000003499 \ldots$ 
28) $\lg$. br. $1^{-\frac{n}{m} \mid 1}=9 \lg m-\lg (m-n)^{9 \mid m}+\left(10-\frac{n}{m}-\frac{1}{2}\right) \lg \frac{10 m-n}{m}+1 \lg 2 \pi$

$-4,33932690130263773 \ldots$.

$+\frac{n}{m} 0,434656033765051676 \ldots$

$+\frac{n^{2}}{m^{2}} 0,000036119334349867 \ldots$

$+\frac{n^{3}}{m^{3}} 0,000003607175470860 \ldots$

$+\frac{n^{4}}{m^{4}} 0,0000003601261098235 \ldots$.

§. 23.

Eine andere Art, den Logarithmen einer Facultät auszudrücken, ist folgende. Nimmt man den Taylor'schen Lehrsatz zu Hülfe, so lässt sich die Function $R\left(\frac{x+n d}{d}\right)$ in (5. §. 21.) in eine Reihe entwickeln, welche nach den Potenzen von $n d$ fortläuft. Es ist nämlich

1) $R\left(\frac{x+n d}{d}\right)=R\left(\frac{x}{d}\right)+\frac{\partial R\left(\frac{x}{d}\right)}{\partial x}-n d+\frac{\partial^{2} R\left(\frac{x}{d}\right)}{1.2 .(\partial x)^{2}} n^{2} d^{2}+\frac{\partial^{3} R\left(\frac{x}{d}\right)}{1.2 .3(\partial x)^{3}} \cdot n^{3} d^{3}$.

Die Lösung der Aufgabe ist demnach darauf zurückgebracht, die Differentiale der Function $R\left(\frac{x}{d}\right)$ zweckmässig darzustellen. Hiezu dient die Glcichung (5. §. 21.) selbst. Sie giebt

$$
R\left(\frac{x}{d}\right)=R\left(\frac{x+k d}{d}\right)-\lg x^{k \mid d} .
$$

Hieraus geht hervor, dass die Darstellung der Differentiale der Function $\boldsymbol{R}\left(\frac{x}{d}\right)$ von dem Exponenten $k$ unabhängig ist; denn der Werth von $R\left(\frac{x}{d}\right)$ bleibt für ein und dasselbe $d$ und $x$ unverändert, wie auch der Exponent k sich ändern mag. Dies gestattet, den Exponenten so anzunchmen, dass sich die Differentiale von $R\left(\frac{x}{d}\right)$ leicht finden lassen; und dies ist der Fall, wenn $k$ unendlich gross angenommen wird, weil alsdann die Glieder in der Function $R\left(\frac{x+k d}{d}\right)$ vom vierten an verschwinden. Setzt man daher $a$ statt $k$, so erhält man 


$$
\text { 2) } \begin{aligned}
\boldsymbol{R}\left(\frac{x}{d}\right)= & \frac{x+\alpha d}{d} \lg (x+\alpha d)-\frac{x+\alpha d}{d}-\frac{\mathrm{L}}{2} \lg (x+\alpha d) \\
& -(\lg x+\lg (x+d)+\lg (x+2 d)+\lg (x+3 d) \ldots
\end{aligned}
$$

Bemerkt man nun, dass

$$
\partial^{p} \lg (x+r d)=(-)^{p-1} \frac{1.2 .3 \ldots .(p-1)}{(x+r d)^{p}}
$$

so ergeben sich aus (2.) die gesuchten Differentiale und es ist

$$
\begin{aligned}
& \frac{\partial_{R}\left(\frac{x}{d}\right)}{\partial x}=\frac{1}{d} \lg (x+a d)-\left(\frac{1}{x}+\frac{1}{x+d}+\frac{1}{x+2 d}+\frac{1}{x+3 d} \ldots\right) \\
& \frac{\partial^{2} R\left(\frac{x}{d}\right)}{1.2(\partial x)^{2}}=+\frac{1}{2}\left(\frac{1}{x^{2}}+\frac{1}{(x+d)^{2}}+\frac{1}{(x+2 d)^{2}}+\frac{1}{(x+3 d)^{2}}+\ldots\right) \\
& \frac{\partial^{3} R\left(\frac{x}{d}\right)}{1.2 .3 .(\partial x)^{3}}=-\frac{1}{3}\left(\frac{1}{x^{3}}+\frac{1}{(x+d)^{3}}+\frac{1}{(x+2 d)^{3}}+\frac{1}{(x+3 d)^{3}}+\ldots\right)
\end{aligned}
$$

u. s. w. Nun ist (S. 16ten Bd. d. Journ. S. 138. §. 127.)

$$
\text { 3) } \begin{aligned}
& \frac{1}{x}+\frac{1}{x+d}+\frac{1}{x+2 d}+\frac{1}{x+3 d}+\ldots \\
= & \frac{1}{d} \lg (x+\alpha d)-\frac{1}{d} \lg x+\frac{1}{2 x}+\frac{d}{2.6 x^{2}}-\frac{d^{3}}{4.30 x^{4}}+\ldots \\
= & \frac{1}{d} \lg (x+\alpha d)+C .
\end{aligned}
$$

Ferner ist (S. 14ten Bd. d. Journ. S. 330. §. 78.)

$$
\text { 4) } \begin{aligned}
& \frac{1}{x^{p}}+\frac{1}{(x+d)^{p}}+\frac{1}{(x+2 d)^{p}}+\frac{1}{(x+3 d)^{p}} \\
& =\frac{1}{(p-1) x^{p-1} d}+\frac{1}{2 x^{p}}+\frac{p d}{2.6 x^{p+1}}-\frac{p^{311} d^{3}}{4.30 x^{p+3}}
\end{aligned}
$$

Diese Gleichungen (3. und 4.) lassen sich auch aus (12. §. 19. und 2. §. 21.) ableiten. Deutet man den Werth von (3.) durch $\Sigma_{0}^{u} \frac{1}{(x+r d)^{p}}$ an, wo für $r$ die Werthe von 0 bis ins Unendliche zu setzen sind, so ergiebt sich aus (5. §. 21.) und aus den hier entwickelten Gleichungen:

5) $\lg x^{n \mid d}=-C n d+\frac{1}{2} n^{2} d^{2} \Sigma_{0}^{\prime \prime} \frac{1}{(x+r d)^{2}}-\frac{1}{3} n^{3} d^{3} \Sigma_{0}^{\alpha} \frac{1}{(x+r d)^{3}}+\frac{1}{4} n^{4} d^{4} \Sigma_{0}^{\prime \prime} \frac{1}{(x+r d)^{4}}-\ldots$.

Diese Gleichung löset die Aufgabe in aller Allgemeinheit auf. Der Exponent kann eine ganze oder gebrochene, positive oder negative Zahl bedeuten. Eben so kann $d$ positiv oder negativ sein. Geht man auf den einfachsten Fall $d=1$ und $x=1$ zurück, was um so mehr geschehen kann, da, wie in 
(§. 11.) gezeigt, jede Facultät auf diese Form zurückgebracht werden kann, so erhält man

6) $\lg 1^{n \mid 1}=-C n+\frac{n^{2}}{2} \Sigma_{1}^{\alpha} \frac{1}{r^{2}}-\frac{n^{3}}{3} \Sigma_{1}^{\prime \prime} \frac{1}{r^{3}}+\frac{n^{4}}{4} \Sigma_{1}^{\alpha} \frac{1}{r^{4}}-\ldots$

Diese Gleichung lässt sich auch noch zweckmässiger umformen. Man kann nämlich in jeder Reihe, welche $\Sigma_{1}^{r} \frac{1}{r^{2}}, \Sigma_{1}^{\alpha} \frac{1}{r^{3}} \ldots$ ausdrückt, das erste Glied ausscheiden. Wird dann in (6.) $x$ statt $r$ gesetzt, so ergiebt sich

$$
\text { 7) } \begin{aligned}
\lg \mathbf{1}^{n \mid 1}=-C n & +\frac{n^{2}}{2}-\frac{n^{3}}{3}+\frac{n^{4}}{4}-\frac{n^{6}}{5}+\ldots \\
& +\frac{n^{2}}{2} \Sigma_{2}^{\alpha} \frac{1}{x^{2}}-\frac{n^{3}}{3} \Sigma_{2}^{\alpha} \frac{1}{x^{3}}+\frac{n^{4}}{4} \Sigma_{2}^{\alpha} \frac{1}{x^{4}}-\ldots
\end{aligned}
$$

Nun ist bekanntlich

$$
\frac{n^{2}}{2}-\frac{n^{3}}{3}+\frac{n^{4}}{4}-\frac{n^{5}}{5}+\ldots=+n-\lg (1+n)
$$

demnach ist aus (7.)

8) $\lg \mathbf{1}^{n \mid 1}=-\lg (1+n)+(1-C) n+\frac{n^{2}}{2} \Sigma_{2}^{\prime \prime} \frac{1}{x^{2}}-\frac{n^{3}}{3} \Sigma_{2}^{\prime \prime} \frac{1}{x^{3}}+\ldots$

Diese Gleichung gilt, wie bemerkt, für jeden Werth von $n$. Sie eignet sich, wie leicht zu sehen, besonders zur Darstellung von Facultäten, deren Exponenten Brüche sind. Man hat dann für die Werthe von $C$ die Constante der harmonischen Reihen und die Summen der reciproken Potenzenreihen zu setzen. Es ergiebt sich

9) $\lg 1^{\frac{n}{m} \mid 1}=-\lg \left(\frac{m+n}{m}\right)^{+}+\frac{n}{m} 0,422784332509846713 \ldots$

$$
\begin{aligned}
& +\frac{1}{2} \frac{n^{2}}{m^{2}} 0,6449340668482264 \ldots \\
& +\frac{1}{3} \frac{n^{3}}{m^{3}} 0,2020569031595943 \ldots \\
& -\frac{1}{4} \frac{n^{4}}{m^{4}} 0,0823232337111382 \ldots
\end{aligned}
$$

Für einen negativen Exponenten ist

10) $\lg 1^{-\frac{n}{m} \mid}=-\lg \left(\frac{m-n}{m}\right)-(1-C) \frac{n}{m}+\frac{n^{2}}{2 m^{2}} \Sigma_{2}^{\alpha} \frac{1}{x^{2}}+\frac{n^{3}}{3 m^{3}} \Sigma_{2}^{\prime \prime} \frac{1}{x^{3}}+\frac{n^{4}}{4 m^{4}} \Sigma_{2}^{\prime \prime \prime} \frac{1}{x^{4}}+\ldots$ 


$$
\begin{aligned}
& \text { 11) } \lg 1^{-\frac{n}{m} \mid}=1-\lg \left(\frac{m-n}{m}\right)-\frac{n}{m} \quad 0,4227843315098467 \\
& +\frac{n^{2}}{2 . m^{2}} 0,6449340668482264 \\
& +\frac{n^{3}}{3 m^{3}} \quad 0,2020569031595943 \\
& +\frac{n^{4}}{4 m^{4}} \quad 0,0823232337111382
\end{aligned}
$$

Die hier entwickelten Formeln lassen sich gut benutzen, wenn $\frac{n}{m}$ ein sehr kleiner Bruch ist, weil dann die Potenzen von $\frac{n}{m}$ schnell convergiren. Ist $\stackrel{n}{m}$ ein Bruch, der sich $\frac{1}{2}$ nähert, so sind viele Rechnungen nöthig, um den Werth von $1^{\frac{n}{m} \mid}$ auf mehrere Decimalstellen genau zu finden. In diesem Falle sind die Ausdrücke des vorigen Paragraph vorzuziehen.

Bei der Darstellung der Werthe von $\lg 1^{\left.\frac{n}{m}\right|^{1}}$ und $\lg \mathbf{1}^{-\left.\frac{n}{m}\right|^{1}}$ sind, wie bemerkt, die Werthe der Summen der reciproken Potenzenreihen nöthig. Euler hat diese Summen (2ter Thl. d. Differenz. Rechnung 6tes Cap. §. 151.) bis zur sechszehnten Potenz und bis auf sechszehn Decimalstellen berechnet. Legendre hat sie (Exercices d. calc. intégr. T. II. Pg. 65.) bis zur 35sten Potenz und ebenfalls bis auf sechzehn Decimalstellen berechnet, weil er fand, dass einige von Euler angegebene Summen nicht richtig sind. Die Unterschiede in den von Beiden mitgetheilten Resultaten kommen bei den Summenausdrücken der 5ten, 7ten, 11ten und 13ten Potenz vor. Um mich von der Zuverlässigkeit der von den beiden Schriftstellern mitgetheilten Resultate zu überzeugen und die richtigere Angabe zu finden, habe ich den Werth der Reihe $\Sigma_{1}^{\prime \prime} \frac{1}{x^{5}}$ nach der (im 14ten Bd. d. Journ. No. 23. S. 330. §. 78.) angegebenen Methode berechnet und folgendes Resultat gefunden:

$$
\Sigma_{1}^{\prime \prime} \frac{1}{x^{5}}=1,036927755143369926 \ldots
$$

Dieses Resultat ist bis zur achtzehnten Stelle richtig und stimmt mit dem von Legendre. Letzterer giebt

$$
\Sigma_{1}^{\alpha} \frac{1}{x^{5}}=1,0369277551433700 .
$$

Euler hat 


$$
\Sigma_{1}^{\alpha} \frac{1}{x^{5}}=1,0369277551068632 ;
$$

was allerdings von dem obigen sehr verschieden ist. Die Unterschiede der Summenausdrücke der 11 ten und 13ten Potenzen in den von Euler und Legendre angegebenen Resultaten sind unbedeutend und scheinen von Druckfehlern herzurühren. Da es nöthig ist, die Werthe der genannten Summenausdrücke zu kennen, so sollen dieselben hieher gesetzt werden. Der Kürze wegen bezeichnen wir sie durch $S_{1}, S_{2}, S_{3} \ldots$ Es ist

$C=0,5772156649015328606 \ldots$

$S_{2}=0,6449340668482264$

$S_{3}=0,2020569031595943$

$S_{4}=0,0823232337111382$

$S_{5}=0,0369277551433699$

$S_{6}=0,0173430619844491$

$S_{7}=0,0083492773810227$

$S_{8}=0,0040773561979443$

$S_{9}=0,0020083928260822$

$S_{10}=0,0009945751278180$

$S_{11}=0,0004941886041194$

$S_{12}=0,0002460865533080$

$S_{13}=0,0001227133475785$

$S_{14}=0,0000612481350587$

$S_{15}=0,0000305882363070$

$S_{16}=0,0000152822594086$

$S_{17}=0,0000076371976379$

$S_{18}=0,0000038172932650$.

$S_{19}=0,0000019082127166$

$S_{20}=0,0000009539620339$

$S_{21}=0,0000004769329868$

$S_{22}=0,0000002384505027$

$S_{23}=0,0000001192199260$

$S_{24}=0,0000000596081891$

$S_{25}=0,0000000298035035$

$S_{26}=0,0000000149015548$

$S_{27}=0,0000000074507118$

$S_{28}=0,0000000037253340$ 


$$
\begin{aligned}
& S_{29}=0,0000000018626597 \\
& S_{30}=0,0000000009313274 \\
& S_{31}=0,0000000004656629 \\
& S_{32}=0,0000000002328312 \\
& S_{33}=0,0000000001164155 \\
& S_{34}=0,0000000000582077 \\
& S_{35}=0,0000000000291038
\end{aligned}
$$

Diese Werthe können für die Rechnung noch zweckmässiger dargestellt werden, wenn man die Division mit den den Gliedern zugehörigen Nennern $\frac{1}{2}, \frac{1}{3}, \frac{1}{4}, \ldots$ ausführt. Dies giebt

$$
\begin{aligned}
& \frac{1}{2} S_{2}=0,3224670334241132 \\
& \frac{1}{3} S_{3}=0,0073523010531981 \\
& \frac{1}{4} S_{4}=0,0205808084277845 \\
& \frac{1}{5} S_{5}=0,0073855510286739 \\
& \frac{1}{6} S_{6}=0,0028905103307415 \\
& \frac{1}{7} S_{7}=0,0011927539117032 \\
& \frac{1}{8} S_{8}=0,0005096695247430 \\
& \frac{1}{9} S_{9}=0,0002231547584535 \\
& \frac{1}{10} S_{10}=0,0000994575127818 \\
& { }_{11}^{11} S_{11}=0,0000449262367381 \\
& \frac{1}{12} S_{12}=0,0000205072127756 \\
& \frac{1}{13} S_{13}=0,0000094394882752 \\
& \frac{1}{14} S_{14}=0,0000043748667899 \\
& \frac{1}{15} S_{15}=0,0000020392157538 \\
& \frac{1}{16} \mathbf{S}_{16}=0,0000009551412130 \\
& \frac{1}{17} S_{17}=0,0000004492469198 \\
& \frac{1}{18} S_{18}=0,0000002120718480 \\
& \frac{1}{19} S_{19}=0,0000001004322482 \\
& \frac{1}{2} S_{20}=0,00000004769810169 \\
& \frac{1}{21} S_{21}=0,0000000227110946 \\
& \frac{1}{22} S_{22}=0,00000001083865921 \\
& \frac{1}{23} S_{23}=0,00000000518347504 \\
& \frac{1}{24} S_{24}=0,00000000248367454 \\
& \frac{1}{25} S_{25}=0,00000000119214014 \\
& \frac{1}{26} \mathbf{S}_{26}=0,00000000057313672 \\
& \frac{1}{27} \mathbf{S}_{27}=0,0000000002759522
\end{aligned}
$$


7. Oettinger, Untersuchungen über die analytischen Facultäten.

$$
\begin{aligned}
& \frac{1}{28} S_{28}=0,00000000013304764 \\
& \frac{1}{29} S_{29}=0,00000000006422964 \\
& \frac{1}{30} S_{30}=0,0000000000310442 \\
& \frac{1}{31} S_{31}=0,0000000000150213 \\
& \frac{1}{32} S_{32}=0,00000000000727597 \\
& \frac{1}{33} S_{33}=0,00000000000352774 \\
& \frac{1}{34} S_{34}=0,0000000000017199 \\
& \frac{1}{35} S_{35}=0,00000000000083154
\end{aligned}
$$

Führt man nun diese Werthe in (9. und 11.) ein, so ergiebt sich:

12) $\lg 1^{\left.\frac{n}{m}\right|^{1}}=-\lg \frac{m+n}{m}+\frac{n}{m} \quad 0,4227843335098467 \ldots$

$$
\begin{aligned}
& +\frac{n^{2}}{m^{2}} \quad 0,3224670334241132 \\
& -\frac{n^{3}}{m^{3}} \quad 0,0673523010531981 \\
& +\frac{n^{4}}{m^{4}} \quad 0,0205808084277845 \\
& -\frac{n^{5}}{m^{6}} \quad 0,00738555102867398 \\
& +\frac{n^{6}}{m^{6}} \quad 0,0028905103307415 \\
& -\frac{n^{7}}{m^{7}} \quad 0,0011927539117032
\end{aligned}
$$

13) $\lg 1^{-\frac{n}{m} \mid 1}=-\lg \left(\frac{m+n}{m}\right)-\frac{n}{m} 0,4227843350984671$

$$
+\frac{n^{2}}{m^{2}} 0,3224670334241132
$$$$
+\frac{n^{3}}{m^{3}} 0,0673523030531981
$$$$
+\frac{n^{4}}{m^{4}} 0,0205808084277845
$$

Diese Ausdrücke gelten für natürliche Logarithmen. Man kann sie in andere für Briggische Logarithmen umformen, wenn man sämmtliche Glieder mit dem Modul 0,4342 $94481 \ldots$ multiplicirt. Dies giebt 
14) lg. br. $1^{\frac{n}{m}} \mid 1=-$ lg. br. $\frac{m+n}{m}+\frac{n}{m} \quad 0,18361290376840$ $+\frac{n^{2}}{m^{2}} 0,1400456532118$ $-\frac{n^{3}}{m^{3}} 0,0292507326917$ $+\frac{n^{4}}{m^{4}} 0,008938131534$ $-\frac{n^{5}}{m^{5}} 0,003207504058$ $+\frac{n^{6}}{m^{6}} 0,001255332686$

$-\frac{n^{7}}{m^{7}} 0,000518006442$ $+\frac{n^{8}}{m^{8}} 0,000221346662$ - $\frac{n^{9}}{m^{9}} 0,000096914880$ $+\frac{n^{10}}{m^{10}} 0,000043193849$ $-\frac{n^{11}}{m^{11}} 0,000019511217$ $+\frac{n^{12}}{m^{12}} 0,000008906169$ - $\frac{n^{18}}{m^{13}} 0,000004099517$ $+\frac{n^{14}}{m^{14}} 0,000001899980$ $-\frac{n^{15}}{m^{15}} 0,000000885620$

15) $\lg$. br. $1^{-\frac{n}{m} \mid}=-\lg \frac{m-n}{m}-\frac{n}{m} 0,18361290376840$ $+\frac{n^{2}}{m^{2}} 0,1400456532118$ $+\frac{n^{3}}{m^{3}} 0,0292507326917$ $+\frac{n^{4}}{m^{4}} 0,008938131534$

Sucht man nun nach den obigen Formeln den natürlichen Logarithmen von $1^{1 b_{0}}$, , so ergiebt sich aus (12.): 
$-\lg 1,01=-0,009950330853168082 \ldots$

Für den Werth der begleitenden Reihe erhält man

$$
K=0,00426002290709843 \ldots
$$

Dies giebt

16) $\lg$ nat. $1^{\frac{1}{100} \mid 1}=-0,00569030794606965 \ldots$.

Aus dem hier und im vorigen Paragraph gefundenen Werthe von $\lg \mathbf{1}^{\mathbf{1}_{0}^{\mathbf{1}} / \mathbf{1}}$ ergiebt sich, dass der Logarithme bis zur sechszehnten Decimalstelle richtig ist.

Benutzt man die Formeln (14. und 15.), welche für gemeine Logarithmen gelten, so zeigt sich, dass die Werthe der Logarithmen bis zur neunten Decimalstelle richtig sich finden lassen. Man erhält aus (14.)

$$
\text { 17) } \lg \text { brigg. } 1^{\frac{1}{10 \sigma} \mid 1}=-0,0024712694 .
$$

Dieser Werth ist bis zur neunten Decimalstelle richtig.

$$
\text { §. } 24 .
$$

$\mathrm{Zu}$ den bisher gegebenen Methoden, den Logarithmen einer Facultät zu berechnen, theilen wir noch folgende mit.

Wird in (3. §. 21.) $x=1, d=1$ gesetzt, so ergiebt sich, mit Rücksicht auf (5. und 14. §. 21.):

$$
\text { 1) } \begin{aligned}
\lg 1^{n \mid 1}= & (n+1) \lg (n+1)-n-1-\frac{1}{2} \lg (n+1)+\frac{1}{2} \lg 2 \pi \\
& +\frac{1}{12(n+1)}-\frac{1}{360(n+1)^{3}}+\frac{1}{1260(n+1)^{6}}-\ldots
\end{aligned}
$$

Bezeichnen wir nun die Reihe rechts durch $S$ und die Vorzahlen ihrer Glieder, wie früher, durch $a_{1}, a_{2}, a_{3} \ldots$ und benutzen das Binomium

$$
\text { 2) } \quad \frac{1}{(x+a)^{m}}=\frac{1}{x^{m}}-[m]_{1} \frac{a}{x^{m+1}}+[m]_{2} \frac{a^{2}}{x^{m+2}}-[m]_{3} \frac{a^{3}}{x^{m+3}}+\ldots
$$

zur Entwicklung der Nenner in $S$, so lässt sich einerseits $x=n$ und $a=1$, und dann $x=1$ und $n=a$ setzen. Im ersten Fall entsteht eine Reihe, deren Glieder nach den fallenden Potenzen von $n$, im andern eine Reihe, deren Glieder nach den steigenden Potenzen geordnet sind. Demnach ist

$$
\text { 3) } \begin{aligned}
& S=\frac{a_{1}}{n}-\frac{a_{1}}{n^{2}}+\frac{a_{1}}{n^{3}}-\frac{a_{1}}{n^{4}} \\
&-\frac{a_{3}}{n^{3}}+[3]_{1} \frac{a_{3}}{n^{4}}-[3]_{2} \frac{a_{3}}{n^{5}}+[3]_{3} \frac{a_{3}}{n^{6}}-[3]_{4} \frac{a_{3}}{n^{7}}+\ldots \\
&+\frac{a_{5}}{n^{5}}-[5]_{1} \frac{a_{5}}{n^{6}}+[5]_{2} \frac{a_{5}}{n^{7}}-\ldots \\
&-\frac{a_{7}}{n^{7}}+\ldots
\end{aligned}
$$


Bezeichnet man die Vorzahl des Iten Gliedes dieser Reihe durch $\boldsymbol{B}_{r}$, so ist die Form dieses Gliedes folgende Reihe:

$$
\text { 4) } B_{r} n^{-r}=(-)^{r-1}\left(a_{1}-[3]_{r-3} a_{3}+[5]_{r-5} a_{5}-[7]_{r-7} a_{7} \ldots\right) n^{-r} \text {. }
$$

Die eingeklammerte Reihe bricht ab, wenn $r-s$ negativ werden sollte. Demnach ergiebt sich folgender entwickelte Ausdruck:

5) $\lg 1^{n \mid 1}=\left(n+\frac{1}{2}\right) \lg (n+1)-(n+1)+\frac{1}{2} \lg 2 \pi$

$$
\begin{aligned}
+a_{1} n^{-1}-a_{1} n^{-2}+\left(a_{1}-a_{3}\right) n^{-3} & -\left(a_{1}-[3]_{1} a_{3}\right) n^{-4} \\
& +\left(a_{1}-[3]_{2} a_{3}+a_{5}\right) n^{-5} \\
& -\left(a_{1}-[3]_{3} a_{3}+[5]_{1} a_{5}\right) n^{-6} \\
& +\left(a_{1}-[3]_{1} a_{3}+[5]_{2} a_{5}-a_{7}\right) n^{-7} \\
& -\left(a_{1}-[3]_{5} a_{3}+[5]_{3} a_{5}-[7]_{1} a_{7}\right) n^{-8}
\end{aligned}
$$

In dieser Gestalt ist die Gleichung nicht brauchbar. Man kann sie aber umändern, wenn man die Werthe für $a_{1}, a_{2}, a_{3} \ldots$. , die in (14. §. 22.) angegeben sind, einführt. Dann ergeben sich für die Vorzahlen der Glieder der begleitenden Reihe folgende Werthe:

$$
\begin{aligned}
& \text { 6) } B_{1}=\frac{1}{12}=0,08333 \ldots \\
& B_{2}=\frac{1}{12}=0,08333 \ldots \\
& B_{3}=\frac{29}{360}=0,080555 \ldots \\
& B_{4}=\frac{3}{40}=0,075 \ldots \\
& B_{5}=\frac{.17}{252}=0,06746031746031 \ldots \\
& B_{6}=\frac{5}{84}=0,05952380952380 \ldots \\
& B_{7}=\frac{89}{1680}=0,0529761904761904 \ldots \\
& B_{8}=\frac{7}{144}=0,0486111 \ldots \\
& B_{9}=\frac{269}{6940}=0,04528619528619 \ldots \\
& B_{10}=\frac{9}{220}=0,040909090 \ldots \\
& B_{11}=\frac{12959}{360360}=0,0359612609612609 \ldots \\
& B_{12}=\frac{11}{312}=0,0352564102564102 \ldots \\
& B_{13}=\frac{43}{1092}=0,0393772893772893 \ldots
\end{aligned}
$$

u. s. w. Aus (5.) ergiebt sich 
7) $\lg \mathbf{1}^{n ! 1}=\left(n+\frac{1}{2}\right) \lg (1+n)-(n+1)+\frac{1}{2} \lg 2 \pi$

$$
+\frac{1}{12 n}-\frac{1}{12 n^{2}}+\frac{29}{360 n^{3}}-\frac{3}{40 n^{4}}+\frac{17}{252 n^{5}}-\frac{5}{84 . n^{6}}+\cdots
$$

Diese Reihe eignet sich besonders zur Berechnung der Werthe der Facultäten von grosser Factorenzahl. Sie wird dieselben um so genauer geben, je grösser $n$ ist. Entwickelt man die Glieder von $S$ in No. 1., indem man in (2.) $x=1, a=n$ setzt, so erhält man

8) $S=a_{1}-a_{1} n+a_{1} n^{2}-a_{1} n^{3}+a_{1} n^{4}-a_{1} n^{5}+\ldots$

$$
\begin{aligned}
& -a_{3}+3 a_{3} n-[3]_{2} a_{3} n^{2}+[3]_{3} a_{3} n^{3}-[3]_{4} a_{3} n^{4}+[3]_{7} a_{3} n^{5}-\ldots \\
& +a_{5}-5 a_{5} n+[5]_{2} a_{5} n^{2}-[5]_{3} a_{5} n^{3}+[5]_{4} a_{5} n^{4}-[5]_{5} a_{5} n^{5}+\ldots \\
& -a_{7}+7 a_{7} n-[7]_{2} a_{7} n^{2}+[7]_{3} a_{7} n^{3}-[7]_{4} a_{7} n^{4}+[8]_{5} a_{7} n^{5}-\ldots
\end{aligned}
$$

Ordnet man nun den Ausdruck (8.) nach den steigenden Potenzen von $n$ und bezeichnet der Kürze wegen die Vorzahlen der Reihe durch $D_{0}, D_{1}, D_{2} \ldots$ so ergiebt sich folgendes Gesetz für das $(r+1)$ te Glied:

$$
\text { 9) } J_{r} n^{r}=(-)^{r}\left(a_{1}-[3]_{r} a_{3}+[5]_{r} a_{5}-[7]_{r} a_{7}+[9]_{r} a_{9}-\ldots .\right) \text {. }
$$

Die Glieder dieser Reihe laufen ins Unendliche fort. Man kann jetzt die Werthe von $a_{1}, a_{2}, a_{3} \ldots$ auf die Bernoullischen Zahlen bringen. Es geschieht durch folgende Bestimmungen nach (13. und 14. §. 22.):

$$
a_{1}=\frac{\mathfrak{A}}{1.2}, \quad a_{3}=\frac{\mathfrak{B}}{3.4}, \quad a_{5}=\frac{\mathfrak{C}}{5.6}, \quad a_{7}=\frac{\mathfrak{D}}{7.8} \ldots,
$$

wo nach Eulers Vorgang $\mathfrak{A}, \mathfrak{B}, \mathfrak{C}, \mathfrak{D}, \ldots$ der Reihe nach die Bernoullischen Zahlen bezeichnen. Benutzt man jetzt die Gleichung (9.), so ergeben sich folgende Ausdrücke:

$$
\begin{aligned}
& \text { 10) } \quad D_{0}=\frac{\mathfrak{A}}{1.2}-\frac{\mathfrak{B}}{3.4}+\frac{\mathfrak{C}}{5.6}-\frac{\mathfrak{D}}{7.8}+\frac{\mathfrak{E}}{9.10}-\ldots \\
& D_{1}=-\left(\frac{\mathfrak{A}}{1.2}-\frac{\mathfrak{B}}{4}+\frac{\mathfrak{C}}{6}-\frac{\mathfrak{D}}{8}+\frac{\mathfrak{E}}{10}-\ldots\right) \\
& D_{2}=\frac{1}{2}(\mathfrak{A}-\mathfrak{B}+\mathfrak{C}-\mathfrak{D}+\mathfrak{E}-\ldots) \\
& J_{3}=-\frac{1}{3}\left(\frac{3 \mathfrak{A}}{2}-\frac{5 \mathfrak{B}}{2}+\frac{7 \mathfrak{C}}{2}-\frac{9 D}{2}+\frac{11 E}{2}-\ldots\right) \\
& D_{4}=\frac{1}{4}\left(\frac{3.4}{2.3} \mathfrak{A}-\frac{5.6}{2.3} \mathfrak{B}+\frac{7.8}{2.3} \mathfrak{C}-\frac{9.10}{2.3} \mathfrak{D}+\frac{11.12}{2.3} \mathfrak{E}-\ldots\right) \\
& n_{5}=-\frac{1}{5}\left(\frac{3.4 .5}{2.3 .4} \mathfrak{A}-\frac{5.6 .7}{2.3 .4} \mathfrak{B}-\frac{7.8 .9}{2.3 .4} \mathfrak{C}-\frac{9.10 .11}{2.3 .4} \mathfrak{D}+\ldots\right)
\end{aligned}
$$

Crelle's Journal f. d. M. Bd. XXXIII. Heft 2. 
Die in Klammern eingeschlossenen Reihen lassen sich summiren, wenn die von Euler (Differ.-Rechn. II. Thl. §. 158. und §. 151., 152.) gegebenen Ausdrücke benutzt werden. Es findet sich

11)

$$
\begin{aligned}
& D_{0}=1-\frac{1}{2} \lg 2 \pi \\
& D_{1} n=-\left(0,5772156 \ldots-1+\frac{1}{2}\right) n=-n .0,5772156 \ldots+n-\frac{1}{2} n \\
& D_{2} n^{2}=\frac{1}{2}\left(0,6449340 \ldots-1+\frac{1}{2}\right) n^{2}=+\frac{n^{2}}{2} 0,6449340 \ldots-\frac{n^{2}}{2}+\frac{1}{2} \cdot \frac{n^{2}}{2} \\
& D_{3} n^{3}=-\frac{1}{3}\left(0,20205690 \ldots-\frac{1}{2}+\frac{1}{2}\right) n^{3}=-\frac{n^{3}}{3} 0,20205690 \ldots+\frac{n^{3}}{2.3}+\frac{1}{2} \cdot \frac{n^{3}}{2} \\
& D_{4} n^{4}=+\frac{1}{4}\left(0,08232323 \ldots-\frac{1}{3}+\frac{1}{2}\right) n^{4}=+\frac{n^{4}}{4} 0,082323 \ldots .+\frac{n^{4}}{3.4}+\frac{1}{2} \cdot \frac{n^{4}}{4} \\
& D_{5} n^{5}=-\frac{1}{5}\left(0,03692775 \ldots-\frac{1}{4}+\frac{1}{2}\right) n^{5}=-\frac{n^{5}}{5} 0,0369275 \ldots .+\frac{n^{5}}{4.5}-\frac{1}{2} \cdot \frac{n^{5}}{5}
\end{aligned}
$$

Diesen Erörterungen zu Folge stellt sich die Gleichung (1.) unter folgender Form dar:

12) $\lg \mathbf{1}^{n \mid 1}=(n+1) \lg (n+1)-n-1-\frac{1}{2} \lg (n+1)+\frac{1}{2} \lg 2 \pi$

$$
+D_{0}+D_{1} n+D_{2} n^{2}+D_{3} n^{3}+D_{4} n^{4}+\ldots
$$

Sollen die Werthe für die Glieder der begleitenden Reihe aus (11. in 12.) eingeführt werden, so sind die zwei Reihen rechts des Gleichheitszeichens in (11.) zu beachten. Sie lassen sich auf folgende Weise kürzer darstellen. Es ist

13)

$$
\begin{aligned}
-\frac{1}{2} \lg (n+1) \quad= & -\frac{1}{2} n+\frac{n^{2}}{2.2}-\frac{n^{3}}{2.3}+\frac{n^{4}}{2.4}-\frac{n^{5}}{2.5}+\ldots \\
-(n+1) \lg (n+1)=-n & +\frac{\cdot n^{2}}{2}-\frac{n^{3}}{3}+\frac{n^{4}}{4}-\frac{n^{5}}{5}+\ldots \\
& \quad-n^{2}+\frac{n^{3}}{2}-\frac{n^{4}}{3}+\frac{n^{5}}{4}-\ldots, \\
= & -n+\frac{n^{2}}{1.2}+\frac{n^{3}}{2.3}-\frac{n^{4}}{3.4}+\frac{n^{5}}{4.5}-\ldots ;
\end{aligned}
$$

folglich ist auch

$$
\text { 14) }-(n+1) \lg (n+1)+2 n=n-\frac{n^{2}}{1.2}+\frac{n^{3}}{2.3}-\frac{n^{4}}{3.4}+\frac{n^{5}}{4.5}-\ldots
$$

Werden nun die Werthe aus (11., 13. und 14. in 12.) eingeführt, so ergiebt sich 
7. Oettinger, Untersuchungen über die analytischen Facultäten.

15) $\lg 1^{n \mid 1}=-\lg (n+1)+(1-0,57721566 \ldots) n$

$$
\begin{aligned}
& +\frac{1}{2} n^{2} \cdot 0,64493406 \ldots \\
& --\frac{n^{3}}{3} \cdot 0,20205690 \ldots \\
& +\frac{n^{4}}{4} \cdot 0,08232323 \ldots
\end{aligned}
$$

Diese Reihe fällt mit der in (\$. 23. 8.) zusammen, weswegen dorthin verwiesen wird.

§. 25.

Wir machen noch eine andere Anwendung von den (in \$. 20.) gefundenen Gleichungen auf die Darstellung der Logarithmen von Facultäten:

Im 14ten Bd. d, Journ. (No. 18. S. 262. §. 72.) ist gezeigt, dass für jede Rieihe, deren Glieder abwechselnde Zeichen haben, folgende Gleichung gilt, wenn $n$ eine gerade Zahl ist:

1) $X_{0}-X_{1}+X_{2}-X_{3} \ldots+X_{n}=\zeta^{-1} X_{n+1}+\zeta^{-1} X_{0}$.

Ist $n$ ungerade, so ist

$$
\text { 2) } X_{0}-X_{1}+X_{2}-X_{3} \ldots-X_{n}=-\zeta^{-1} X_{n+1}+\zeta^{-1} X_{0} \text {. }
$$

In ( $\left(\right.$. 20.) ist gezeigt worden, wie die Functionen $\zeta^{-1} X_{n+1}, \zeta^{-1} X_{0}$ entwickelt werden. Führt man die angezeigten Operationen aus, so erhält man

3) $\lg \frac{x^{n \mid 2 d}}{(x+d)^{n-1 \mid 2 d}}=\frac{1}{2} \lg (x+2 n d)+\frac{d}{4(x+2 n d)}-\frac{d^{3}}{8.3 .(x+2 n d)^{3}}+\frac{d^{5}}{4.5 .(x+2 n d)^{5}}-\ldots$

$$
+\frac{1}{2} \lg x-\frac{d}{4 x}+\frac{d^{3}}{8.3 x^{3}}-\frac{d^{5}}{4.5 x^{5}}+\frac{17 d^{7}}{16.7 x^{7}}-\ldots
$$

4) $\lg \frac{x^{n \mid 2 d}}{(x+d)^{n \mid 2 d}}=-\frac{1}{2} \lg (x+2 n d)-\frac{d}{4(x+2 n d)}+\frac{d^{3}}{8.3 \cdot(x+2 n d)^{3}}-\frac{d^{5}}{4.5 .(x+2 n d)^{5}}+\ldots$

$$
+\frac{1}{2} \lg x-\frac{d}{4 x}+\frac{d^{3}}{8 . \overline{3 x^{3}}}-\frac{d^{5}}{4.5 x}+\frac{17 d^{7}}{16.7 . d^{7}}-\ldots
$$

Wir setzen auch hier, wie in (\$. 21.), statt der beiden ins Unendliche fortlaufenden Reihen einfachere und ähnliche Zeichen und drücken die Gleichungen auf folgende Weise aus:
5) $\lg \frac{x^{n \mid 2 d}}{(x+d)^{n-1 \mid 2 d}}=+Q\left(\frac{x+2 n d}{d}\right)+S\left(\frac{x}{d}\right)$,
6) $\lg \frac{x^{n \mid 2 d}}{(x+d)^{n \mid 2 d}}=-Q\left(\frac{x+2 n d}{d}\right)+S\left(\frac{x}{d}\right)$. 
Geht man nun wieder auf einfachere Fälle über und setzt $d=1, x=1$, so ergiebt sich aus (3. und 4.)

7) $\lg \frac{1^{n \mid 2}}{2^{n-1 \mid 1}}=\frac{1}{2} \lg (2 n+1)+\frac{1}{4(2 n+1)}-\frac{1}{24(2 n+1)^{3}}+\frac{1}{20(2 n+1)^{5}}-\ldots$

$$
-\frac{1}{4}+\frac{1}{24}-\frac{1}{20}+\frac{17}{112}-\ldots
$$

8) $\lg \frac{1^{n \mid 2}}{2^{n \mid 2}}=-\frac{1}{2} \lg (2 n+1)-\frac{1}{4(2 n+1)}+\frac{1}{24(2 n+1)^{3}}-\frac{1}{20(2 n+1)^{5}}+\ldots$

$$
-\frac{1}{4}+\frac{1}{24}-\frac{2}{20}+\frac{17}{112}-\ldots
$$

Es handelt sich jetzt wieder um die Werthbestimmung der Function $S(1)$. Aus (29. §. 13, ) erhält man für ein unendlich-grosses $n$ :

$$
\text { 9) } \frac{\mathbf{1}_{|2|}^{n}}{2^{n-1 \mid 1}}=2 V^{\frac{\bar{n}}{\pi}} \text {. }
$$

Wird in (7.) $n$ gleichfalls unendlich-gross gesetzt, so findet sich

$$
\text { 10) } \frac{1^{n \mid 2}}{2^{n-1 \mid 1}}=\frac{1}{2} \lg (2 n+1)+S(1) \text {. }
$$

Nimmt man von 9 den Logarithmen, so erhält man aus (9. und 10.):

$$
\lg 2+\frac{1}{2} \lg n-\frac{1}{2} \lg \pi=\frac{1}{2} \lg (2 n+1)+S(1) .
$$

Da nun bei unendlich-grossem $n$ der Ausdruck $\frac{1}{2} \lg (2 n+1)$ in $\frac{1}{2} \lg 2 n$ übergeht, so ergiebt sich

$$
\text { 11) } S(1)=\frac{1}{2} \lg \frac{2}{\pi} \text {. }
$$

Der nämliche Werth lässt sich auch aus (8.) ableiten. Es ist

12) $\lg \frac{1^{n \mid 2}}{2^{n-1 \mid 1}}=\frac{1}{2} \lg (2 n+1)+\frac{1}{2} \lg \frac{2}{\pi}+\frac{1}{4(2 n+1)}-\frac{1}{24(2 n+1)^{3}}+\frac{1}{20(2 n+1)^{5}}-\ldots$

13) $\lg \frac{1^{n \mid 2}}{2^{n \mid 2}}=-\frac{1}{2} \lg (2 n+1)+\frac{1}{2} \lg \frac{2}{\pi}-\frac{1}{4(2 n+1)}+\frac{1}{24(2 n+1)^{3}}-\frac{1}{20(2 n+1)^{5}}+\ldots$

Diese Gleichungen lassen sich leicht zu weitern Ableitungen benutzen. Es ist z. B. bekanntlich

$$
\begin{aligned}
\frac{2 n(2 n-1)(2 n-2) \ldots .(n+1)}{1.2 .3 \ldots n} & =\frac{2 n(2 n-1) \ldots 3.2 .1}{1.2 .3 \ldots n .1 .2 .3 \ldots . . n} \\
& =\frac{1.3 .5 \ldots .(2 n-1) .2 .4 .6 \ldots 2 n}{1.2 .3 \ldots n .1 .2 .3 \ldots . . n} \\
& =\frac{1^{n \mid 2} \cdot 2^{n}}{1^{n \mid 1}}
\end{aligned}
$$


Wird hier Zähler und Nenner mit $2^{n}$ multiplicirt, so ergiebt sich

$$
\text { 14) } \frac{2 n(2 n-1 \ldots .(n+1)}{1.2 \ldots . . n}=\frac{1^{n \mid 2} \cdot 2^{n} \cdot 2^{n}}{2^{n \mid 2}} \text {. }
$$

Verbindet man (13.) mit (14.), so folgt

15) $\lg \frac{(2 n)^{n \mid-1}}{1^{n \mid 1}}=\lg \frac{\mathbf{1}^{n \mid 2}}{2^{n \mid 2}}+2 n \lg 2$

$$
=2 n \lg 2-\frac{1}{2} \lg (2 n+1)+\frac{1}{2} \lg \frac{2}{\pi}-\frac{1}{4(2 n+1)}+\frac{1}{24(2 n+1)^{3}}-\frac{1}{20(2 n+1)^{5}}+\ldots
$$

und da bekanntlich

$$
(2 n)_{n}=1+n . n+(n)_{2}(n)_{2}+(n)_{3}(n)_{3}+(n)_{4}(n)_{4}+\ldots+1
$$

ist, so hat man ferner

16

$$
\begin{aligned}
& \lg \left[1+n^{2}+(n)_{2}(n)_{2}+(n)_{3}(n)_{3}+(n)_{4}(n)_{4}+\ldots+1\right] \\
& =\left(2 n+\frac{1}{2}\right) \lg 2-\frac{1}{2} \lg (2 n+1)-\lg \pi-\frac{1}{4(2 n+1)}+\frac{1}{24(2 n+1)^{3}}-\frac{1}{20(2 n+1)^{5}}
\end{aligned}
$$

(Die Fortsetzung folgt.) 